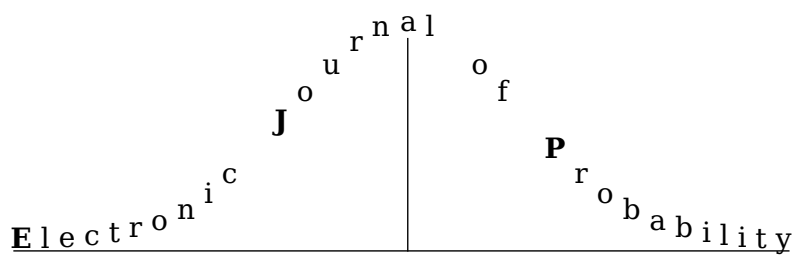

Electron. J. Probab. 26 (2021), article no. 20, 1-25.

ISSN: 1083-6489 https://doi.org/10.1214/21-EJP580

\title{
Conditional propagation of chaos for mean field systems of interacting neurons*
}

\author{
Xavier Erny $^{\dagger} \quad$ Eva Löcherbach ${ }^{\ddagger} \quad$ Dasha Loukianova $^{\dagger}$
}

\begin{abstract}
We study the stochastic system of interacting neurons introduced in [5] and in [10] in a diffusive scaling. The system consists of $N$ neurons, each spiking randomly with rate depending on its membrane potential. At its spiking time, the potential of the spiking neuron is reset to 0 and all other neurons receive an additional amount of potential which is a centred random variable of order $1 / \sqrt{N}$. In between successive spikes, each neuron's potential follows a deterministic flow. We prove the convergence of the system, as $N \rightarrow \infty$, to a limit nonlinear jumping stochastic differential equation driven by Poisson random measure and an additional Brownian motion $W$ which is created by the central limit theorem. This Brownian motion is underlying each particle's motion and induces a common noise factor for all neurons in the limit system. Conditionally on $W$, the different neurons are independent in the limit system. This is the conditional propagation of chaos property. We prove the well-posedness of the limit equation by adapting the ideas of [12] to our frame. To prove the convergence in distribution of the finite system to the limit system, we introduce a new martingale problem that is well suited for our framework. The uniqueness of the limit is deduced from the exchangeability of the underlying system.
\end{abstract}

Keywords: Mean field interaction; piecewise deterministic Markov processes; interacting particle systems; propagation of chaos; exchangeability; Hewitt Savage theorem; martingale problem; empirical measure.

MSC2020 subject classifications: 60J76; 60K35; 60G55; 60G09.

Submitted to EJP on March 2, 2020, final version accepted on January 10, 2021.

Supersedes arXiv: 1909.02925.

Supersedes HAL: hal - 02280882.

*This article was first posted with an error in the code that has produced the graphics of the figure 1 . There was also a mistake in the caption (the arctan is missing). The errors were corrected on 27 May 2021.

†Université Paris-Saclay, CNRS, Univ Evry, Laboratoire de Mathématiques et Modélisation d’Evry, 91037, Evry, France. E-mail: xavier .erny@univ-evry.fr E-mail: dasha. loukianova@univ-evry.fr

${ }^{\ddagger}$ Statistique, Analyse et Modélisation Multidisciplinaire, Université Paris 1 Panthéon-Sorbonne, EA 4543 et FR FP2M 2036 CNRS. E-mail: eva. locherbach@univ-paris1.fr 


\section{Introduction}

This paper is devoted to the study of the Markov process $X_{t}^{N}=\left(X_{t}^{N, 1}, \ldots, X_{t}^{N, N}\right)$ taking values in $\mathbb{R}^{N}$ and having generator $A^{N}$ which is defined for any smooth test function $\varphi: \mathbb{R}^{N} \rightarrow \mathbb{R}$ by

$$
A^{N} \varphi(x)=-\alpha \sum_{i=1}^{N} \partial_{x^{i}} \varphi(x) x^{i}+\sum_{i=1}^{N} f\left(x^{i}\right) \int_{\mathbb{R}} \nu(d u)\left(\varphi\left(x-x^{i} e_{i}+\sum_{j \neq i} \frac{u}{\sqrt{N}} e_{j}\right)-\varphi(x)\right),
$$

where $x=\left(x^{1}, \ldots, x^{N}\right)$ and where $e_{j}$ denotes the $j$-th unit vector in $\mathbb{R}^{N}$. In the above formula, $\alpha>0$ is a fixed parameter and $\nu$ is a centred probability measure on $\mathbb{R}$ having a second moment.

Informally, the process $\left(X^{N, j}\right)_{1 \leq j \leq N}$ solves

$$
X_{t}^{N, i}=X_{0}^{N, i}-\alpha \int_{0}^{t} X_{s}^{N, i} d s-\int_{0}^{t} X_{s-}^{N, i} d Z_{s}^{N, i}+\frac{1}{\sqrt{N}} \sum_{j \neq i} \int_{0}^{t} U^{j}(s) d Z_{s}^{N, j},
$$

where the $U^{j}(s)$ are i.i.d. centred random variables distributed according to $\nu$, and where for each $1 \leq j \leq N, Z^{N, j}$ is a simple counting process on $\mathbb{R}_{+}$having stochastic intensity $s \mapsto f\left(X_{s-}^{N, j}\right)$.

The particle system (1.1) is a version of the model of interacting neurons considered in [5], inspired by [11], and then further studied in [10] and [4]. The system consists of $N$ interacting neurons. In (1.1), $Z_{t}^{N, j}$ represents the number of spikes emitted by the neuron $j$ in the interval $[0, t]$ and $X_{t}^{N, j}$ the membrane potential of the neuron $j$ at time $t$. Spiking occurs randomly following a point process of rate $f(x)$ for any neuron of which the membrane potential equals $x$. Each time a neuron emits a spike, the potentials of all other neurons receive an additional amount of potential. In [5], [10] and [4] this amount is of order $N^{-1}$, leading to classical mean field limits as $N \rightarrow \infty$. On the contrary to this, in the present article we study a diffusive scaling where all neurons $j$ receive the same random quantity $U / \sqrt{N}$ at spike times $t$ of neuron $i, i \neq j$. The random variable $U$ is centred modeling the fact that the synaptic weights are balanced. Moreover, right after its spike, the potential of the spiking neuron $i$ is reset to 0 , interpreted as resting potential. Finally, in between successive spikes, each neuron has a loss of potential of rate $\alpha$.

Before introducing the exact limit equation for the system (1.1), let us explain informally how the limit particle system associated to $\left(X^{N, i}\right)_{1<i<N}$ should a priori look like. Suppose for the moment that we already know that there exists a process $\left(\bar{X}^{1}, \bar{X}^{2}, \bar{X}^{3}, \ldots\right) \in D\left(\mathbb{R}_{+}, \mathbb{R}\right)^{\mathbb{N}^{*}}$ such that for all $K>0, \mathcal{L}\left(X^{N, 1}, \ldots, X^{N, K}\right)$ converges weakly to $\mathcal{L}\left(\bar{X}^{1}, \ldots, \bar{X}^{K}\right)$ in $D\left(\mathbb{R}_{+}, \mathbb{R}\right)^{K}$, as $N \rightarrow \infty$, holds. In equation (1.1) the only term that depends on $N$ is the martingale term which is approximately given by

$$
M_{t}^{N}=\frac{1}{\sqrt{N}} \sum_{j=1}^{N} \int_{0}^{t} U^{j}(s) d Z_{s}^{N, j} .
$$

Then in the infinite neuron model, each process $\bar{X}^{i}$ should solve the equation (1.1), where the term $M_{t}^{N}$ is replaced by $M_{t}:=\lim _{N \rightarrow \infty} M_{t}^{N}$. Because of the scaling in $N^{-1 / 2}$, the limit martingale $M_{t}$ will be a stochastic integral with respect to some Brownian motion, and its variance the limit of

$$
\mathbb{E}\left[\left(M_{t}^{N}\right)^{2}\right]=\sigma^{2} \int_{0}^{t} \mathbb{E}\left[\frac{1}{N} \sum_{j=1}^{N} f\left(X_{s}^{N, j}\right)\right] d s
$$


where $\sigma^{2}$ is the variance of any of the $U^{j}(s)$. Therefore, the limit martingale must be of the form

$$
M_{t}=\sigma \int_{0}^{t} \sqrt{\lim _{N \rightarrow \infty} \frac{1}{N} \sum_{j=1}^{N} f\left(X_{s}^{N, j}\right)} d W_{s}=\sigma \int_{0}^{t} \sqrt{\lim _{N \rightarrow \infty} \mu_{s}^{N}(f)} d W_{s},
$$

where $\mu_{s}^{N}$ is the empirical measure of the system $\left(X_{s}^{N, j}\right)_{1 \leq j \leq N}$ and $W$ is a one-dimensional standard Brownian motion.

Since the law of the $N$-particle system $\left(X^{N, 1}, \ldots, X^{N, N}\right)$ is symmetric, the law of the limit system $\bar{X}=\left(\bar{X}^{1}, \bar{X}^{2}, \bar{X}^{3}, \ldots\right)$ must be exchangeable, that is, for all finite permutations $\sigma$, we have that $\mathcal{L}\left(\bar{X}^{\sigma(1)}, \bar{X}^{\sigma(2)}, \ldots\right)=\mathcal{L}(\bar{X})$. In particular, the theorem of Hewitt-Savage, see [13], implies that the random limit

$$
\mu_{s}:=\lim _{N \rightarrow \infty} \frac{1}{N} \sum_{i=1}^{N} \delta_{\bar{X}_{s}^{i}}
$$

exists. Supposing that $\mu_{s}^{N}$ converges, it necessarily converges towards $\mu_{s}$. Therefore, $\bar{X}$ should solve the limit system

$$
\bar{X}_{t}^{i}=\bar{X}_{0}^{i}-\alpha \int_{0}^{t} \bar{X}_{s}^{i} d s-\int_{0}^{t} \bar{X}_{s-}^{i} d \bar{Z}_{s}^{i}+\sigma \int_{0}^{t} \sqrt{\mu_{s}(f)} d W_{s}, i \in \mathbb{N},
$$

where each $\bar{Z}^{i}$ has intensity $t \mapsto f\left(\bar{X}_{t-}^{i}\right)$, and where $\mu_{s}$ is given by (1.2). The above arguments are made rigorous in Sections 3.1 and 3.2 below.

Let us briefly discuss the form of the limit equation (1.3). As we have already observed in a different framework in our previous paper [9], the scaling in $N^{-1 / 2}$ in (1.1) creates a Brownian motion $W$ in the limit system (1.3). We will show that the presence of this Brownian motion entails a conditional propagation of chaos property, that is the conditional independence of the particles given $W$. In particular, the limit measure $\mu_{s}$ will be random. This differs from the classical framework, where the scaling is in $N^{-1}$ (see e.g. [6], [8] in the framework of Hawkes processes, and [5], [10] and [4] in the framework of systems of interacting neurons), leading to a deterministic limit measure $\mu_{s}$ and the true propagation of chaos property implying that the particles of the limit system are independent.

This is not the first time that conditional propagation of chaos is studied in the literature; it has already been considered e.g. in [2], [3] and [7]. But in these papers the common noise, represented by a common (maybe infinite dimensional) Brownian motion, is already present at the level of the finite particle system, the mean field interactions act on the drift of each particle, and the scaling is the classical one in $N^{-1}$. On the contrary to this, in our model, this common Brownian motion is only present in the limit, and it is created by the central limit theorem as a consequence of the joint action of the small jumps of the finite size particle system. Moreover, in our model, the interactions survive as a variance term in the limit system due to the diffusive scaling in $N^{-1 / 2}$.

Now let us discuss the form of the random measure $\mu_{s}$ appearing in (1.2). The theorem of Hewitt-Savage, [13], implies that the law of $\left(\bar{X}_{s}^{i}\right)_{i>1}$ is a mixture directed by the law of $\mu_{s}$. As it has been remarked by [2] and [3], this conditioning reflects the dependencies between the particles.

We will show that the variables $\bar{X}^{i}$ are conditionally independent given the Brownian motion $W$. As a consequence, $\mu_{s}$ is necessarily given by the conditional law of the solution given the Brownian motion, that is, $P$-almost surely,

$$
\mu_{s}(\cdot)=P\left(\bar{X}_{s}^{i} \in \cdot\left(W_{t}\right)_{0 \leq t \leq s}\right)=P\left(\bar{X}_{s}^{i} \in \cdot \mid W\right),
$$


where the above definition does not depend on $i \in \mathbb{N}$ by exchangeability. Equation (1.3) together with (1.4) gives a precise definition of the limit system.

The nonlinear SDE (1.3) is not clearly well-posed, and our first main result, Theorem 2.6, gives appropriate conditions on the system that guarantee pathwise uniqueness and the existence of a unique strong solution to (1.3).

We then prove, in Sections 3.1 and 3.2, our main Theorem 2.13 stating the convergence in distribution of the sequence of empirical measures $\mu^{N}=N^{-1} \sum_{i=1}^{N} \delta_{\left(X_{t}^{N, i}\right)_{t \geq 0}}$, in $\mathcal{P}\left(D\left(\mathbb{R}_{+}, \mathbb{R}\right)\right)$, to the random limit $\mu=P\left(\left(\bar{X}_{t}^{i}\right)_{t \geq 0} \in \cdot W\right)$.

To do so, we first prove that under suitable conditions on the parameters of the system, the sequence $\mu^{N}$ is tight. We then follow a classical road and identify every possible limit as solution of a martingale problem. Since the random limit measure $\mu$ will only be the directing measure of the limit system (that is, the conditional law of each coordinate, but not its law), this martingale problem is not a classical one. It is in particular designed to reflect the correlations between the particles and to describe all possible limits of couples of neurons. To identify $\mu$ as conditional law knowing $W$, that is, to prove that the only common randomness is the one present in the driving Brownian motion $W$, we introduce an auxiliary particle system which is a mean field particle version of the limit system, constructed with the same underlying Brownian motion, and we provide an explicit control on the distance between the two systems.

Organisation of the paper. In Section 2, we state the assumptions, and formulate the main results on the well-posedness of the limit system, Theorems 2.6 and 2.11. Section 3 is devoted to the proof of the convergence of $\mu^{N}:=\sum_{j=1}^{N} \delta_{X^{N, j}}$ (Theorem 2.13). In particular, we introduce our new martingale problem in Section 3.2 and prove the uniqueness of the limit law in Theorem 3.4. Finally, some of our proofs are gathered in Section 4 .

\section{Notation, model and main results}

\subsection{Notation}

We use the following notation throughout the paper. If $E$ is a metric space, we note $\mathcal{P}(E)$ the space of probability measures on $E$ endowed with the topology of the weak convergence.

For any $n, p \in \mathbb{N}^{*}$, we note $C_{b}^{n}\left(\mathbb{R}^{p}\right)$ (resp. $C_{b}^{n}\left(\mathbb{R}^{p}, \mathbb{R}_{+}\right)$) the set of real-valued functions $g$ (resp. non-negative functions $g$ ) defined on $\mathbb{R}^{p}$ which are $n$ times continuously differentiable such that $g^{(k)}$ is bounded for each $0 \leq k \leq n$, and $C_{c}^{n}\left(\mathbb{R}^{p}\right)$ the set of real-valued functions $g \in C_{b}^{n}\left(\mathbb{R}^{p}\right)$ that have a compact support.

In addition, in what follows $D\left(\mathbb{R}_{+}, \mathbb{R}\right)$ denotes the space of càdlàg functions from $\mathbb{R}_{+}$to $\mathbb{R}$, endowed with the Skorokhod metric, and $C$ and $K$ denote arbitrary positive constants whose values can change from line to line in an equation. We write $C_{\theta}$ and $K_{\theta}$ if the constants depend on some parameter $\theta$.

\subsection{The finite system}

We consider, for each $N \geq 1$, a family of i.i.d. Poisson measures $\left(\pi^{i}(d s, d z, d u)\right)_{i=1, \ldots, N}$ on $\mathbb{R}_{+} \times \mathbb{R}_{+} \times \mathbb{R}$ having intensity measure $d s d z \nu(d u)$ where $\nu$ is a probability measure on $\mathbb{R}$, as well as an i.i.d. family $\left(X_{0}^{N, i}\right)_{i=1, \ldots, N}$ of $\mathbb{R}$-valued random variables independent of the Poisson measures. The object of this paper is to study the convergence of the Markov process $X_{t}^{N}=\left(X_{t}^{N, 1}, \ldots, X_{t}^{N, N}\right)$ taking values in $\mathbb{R}^{N}$ and solving, for $i=1, \ldots, N$, 
for $t \geq 0$,

$$
\left\{\begin{aligned}
X_{t}^{N, i}= & X_{0}^{N, i}-\alpha \int_{0}^{t} X_{s}^{N, i} d s-\int_{[0, t] \times \mathbb{R}_{+} \times \mathbb{R}} X_{s-}^{N, i} \mathbb{1}_{\left\{z \leq f\left(X_{s-}^{N, i}\right)\right\}} \pi^{i}(d s, d z, d u) \\
& +\frac{1}{\sqrt{N}} \sum_{j \neq i} \int_{[0, t] \times \mathbb{R}_{+} \times \mathbb{R}} u \mathbb{1}_{\left\{z \leq f\left(X_{s-}^{N, j}\right)\right\}} \pi^{j}(d s, d z, d u), \\
X_{0}^{N, i} \sim & \nu_{0} .
\end{aligned}\right.
$$

The coefficients of this system are the exponential loss factor $\alpha>0$, the jump rate function $f: \mathbb{R} \mapsto \mathbb{R}_{+}$and the probability measures $\nu$ and $\nu_{0}$.

In order to guarantee existence and uniqueness of a strong solution of (2.1), we introduce the following hypothesis.

Assumption 2.1. The function $f$ is Lipschitz continuous.

In addition, we also need the following condition to obtain a priori bounds on some moments of the process $\left(X^{N, i}\right)_{1 \leq i \leq N}$.

Assumption 2.2. Assume that $\int_{\mathbb{R}} x d \nu(x)=0, \int_{\mathbb{R}} x^{2} d \nu(x)<+\infty$, and $\int_{\mathbb{R}} x^{2} d \nu_{0}(x)<+\infty$.

Under Assumptions 2.1 and 2.2, existence and uniqueness of a strong solution of (2.1) follow from Theorem IV.9.1 of [14], exactly in the same way as in Proposition 6.6 of [9].

We now define precisely the limit system and discuss its properties before proving the convergence of the finite to the limit system.

\subsection{The limit system}

The limit system $\left(\bar{X}^{i}\right)_{i \geq 1}$ is an exchangeable system given by

$$
\left\{\begin{aligned}
\bar{X}_{t}^{i}= & \bar{X}_{0}^{i}-\alpha \int_{0}^{t} \bar{X}_{s}^{i} d s-\int_{[0, t] \times \mathbb{R}_{+}} \bar{X}_{s-}^{i} \mathbb{1}_{\left\{z \leq f\left(\bar{X}_{s-}^{i}\right)\right\}} \pi^{i}(d s, d z) \\
& +\sigma \int_{0}^{t} \sqrt{\mathbb{E}\left[f\left(\bar{X}_{s}^{i}\right) \mid \mathcal{W}\right]} d W_{s} \\
\bar{X}_{0}^{i} \sim & \nu_{0} .
\end{aligned}\right.
$$

In the above equation, $\left(W_{t}\right)_{t \geq 0}$ is a standard one-dimensional Brownian motion, $\pi^{i}$ $(i \geq 1)$ are independent Poisson random measures on $\mathbb{R}_{+}^{2}$ having intensity $d t \cdot d z$ that are independent of $W$, and $\mathcal{W}=\sigma\left\{W_{t}, t \geq 0\right\}$. Moreover, the initial positions $\bar{X}_{0}^{i}, i \geq 1$, are i.i.d., independent of $W$ and of the Poisson random measures, distributed according to $\nu_{0}$ which is the same probability measure as in (2.1).

The limit equation (2.2) is not clearly well-posed and requires additional conditions. Let us briefly comment on the type of difficulties that one encounters when dealing with (2.2). Roughly speaking, the jump terms demand to work in an $L^{1}$-framework, while the diffusive terms demand to work in an $L^{2}$-framework. [12] proposes a unified approach to deal both with jump and with diffusion terms in a non-linear framework, and we shall rely on his ideas in the sequel. The presence of the random volatility term which involves conditional expectation causes however additional technical difficulties. Finally, another difficulty comes from the fact that the jumps induce non-Lipschitz terms of the form $\bar{X}_{s}^{i} f\left(\bar{X}_{s}^{i}\right)$. For this reason a classical Wasserstein-1-coupling is not appropriate for the jump terms. Therefore we propose a different distance which is inspired by the one already used in [10]. To do so, we need to work under the following additional assumption.

Assumption 2.3. 1. We suppose that inf $f>0$.

2. There exists a function $a \in C_{b}^{2}\left(\mathbb{R}, \mathbb{R}_{+}\right)$, strictly increasing, such that, for some constant $C$, for all $x, y \in \mathbb{R}$,

$$
\left|a^{\prime \prime}(x)-a^{\prime \prime}(y)\right|+\left|a^{\prime}(x)-a^{\prime}(y)\right|+\left|x a^{\prime}(x)-y a^{\prime}(y)\right|+|f(x)-f(y)| \leq C|a(x)-a(y)| .
$$


Note that Assumption 2.3 implies Assumption 2.1 as well as the boundedness of the rate function $f$.

Remark 2.4. Notice that $f$ lowerbounded implies that the application $\mathcal{P}(\mathbb{R}) \ni \nu \mapsto$ $g(\nu):=\sqrt{\nu(f)}$ is Lipschitz when considering the Wasserstein-1-distance on $\mathcal{P}(\mathbb{R})$.

Let us give some examples of functions $f$ that satisfy Assumption 2.3.

Example 2.5. Any positive bounded and lowerbounded function $f \in C_{b}^{1}\left(\mathbb{R}, \mathbb{R}_{+}\right)$with

$$
\left|f^{\prime}(x)\right| \leq \frac{C}{(1+|x|)^{1+\varepsilon}}
$$

for all $x \in \mathbb{R}$, where $C$ and $\varepsilon$ are some positive constants, satisfies Assumption 2.3 with

$$
a=\int_{-\infty}^{x} \frac{d y}{(1+\psi(y))^{1+\varepsilon}},
$$

where $\psi$ is any smooth non-negative function satisfying $\psi(y)=|y|$ for $|y| \geq 1$. If (2.3) holds with $\varepsilon=1$, then we may choose simply $a(x)=\arctan (x)+\pi / 2$.

Finally, fix some $-\infty<a<b<\infty$. Then any function $f \in C_{b}^{1}\left(\mathbb{R}, \mathbb{R}_{+}\right)$which is constant below $a$ and above $b$ satisfies Assumption (2.3).

Let us note that this kind of function is interesting from a neuroscience point of view, if it is in addition non decreasing. Indeed, when the potential of a neuron is below $a$ (resp. above $b$ ), its spiking rate is minimal (resp. maximal), such that the neuron can be considered as inactive (resp. active).

Under these additional assumptions we obtain the well-posedness of each coordinate of the limit system (2.2), that is, of the $\left(\mathcal{F}_{t}\right)_{t}$ - adapted process $\left(\bar{X}_{t}\right)_{t}$ having càdlàg trajectories which is solution of the SDE

$$
\left\{\begin{aligned}
d \bar{X}_{t} & =-\alpha \bar{X}_{t} d t-\bar{X}_{t-} \int_{\mathbb{R}_{+}} \mathbb{1}_{\left\{z \leq f\left(\bar{X}_{t-}\right)\right\}} \pi(d t, d z)+\sigma \sqrt{\mu_{t}(f)} d W_{t} \\
\bar{X}_{0} & \sim \nu_{0}, \quad \mu_{t}(f)=\mathbb{E}\left[f\left(\bar{X}_{t}\right) \mid \mathcal{W}\right]=\mathbb{E}\left[f\left(\bar{X}_{t}\right) \mid \mathcal{W}_{t}\right] .
\end{aligned}\right.
$$

Here, $\mathcal{F}_{t}=\sigma\left\{\pi([0, s] \times A), s \leq t, A \in \mathcal{B}\left(\mathbb{R}_{+} \times \mathbb{R}\right)\right\} \vee \mathcal{W}_{t}, \mathcal{W}_{t}=\sigma\left\{W_{s}, s \leq t\right\}$ and $\mathcal{W}=$ $\sigma\left\{W_{s}, s \geq 0\right\}$.

Theorem 2.6. Grant Assumption 2.3.

1. Pathwise uniqueness holds for the nonlinear SDE (2.4).

2. If additionally, $\int_{\mathbb{R}} x^{2} d \nu_{0}(x)<+\infty$, then there exists a unique strong solution $\left(\bar{X}_{t}\right)_{t \geq 0}$ of the nonlinear SDE (2.4), which is $\left(\mathcal{F}_{t}\right)_{t}$ - adapted with càdlàg trajectories, satisfying for every $t>0$,

$$
\mathbb{E}\left[\sup _{0 \leq s \leq t} \bar{X}_{s}^{2}\right]<+\infty
$$

Remark 2.7. Notice that the stochastic integral $\int_{0}^{t} \sqrt{\mu_{s}(f)} d W_{s}$ is well-defined since $s \mapsto \sqrt{\mu_{s}(f)}$ is an $\left(\mathcal{W}_{t}\right)_{t}$-progressively measurable process.

We now given the proof of Item 1. of the above theorem. The proof of Item 2. which follows a classical Picard iteration is postponed to Section 4.

Proof of Item 1. of Theorem 2.6. Consider two strong solutions $\left(\hat{X}_{t}\right)_{t \geq 0}$ and $\left(\check{X}_{t}\right)_{t \geq 0}$, $\left(\mathcal{F}_{t}\right)_{t}$-adapted, defined on the same probability space and driven by the same Poisson random measure $\pi$ and the same Brownian motion $W$, and with $\hat{X}_{0}=\check{X}_{0}$. We consider $Z_{t}:=a\left(\hat{X}_{t}\right)-a\left(\check{X}_{t}\right)$. Denote $\hat{\mu}_{s}(f)=\mathbb{E}\left[f\left(\hat{X}_{s}\right) \mid \mathcal{W}_{s}\right]$ and $\check{\mu}_{s}(f)=\mathbb{E}\left[f\left(\check{X}_{s}\right) \mid \mathcal{W}_{s}\right]$. 


\section{Conditional propagation of chaos}

Using Itô's formula, we can write

$$
\begin{gathered}
Z_{t}=-\alpha \int_{0}^{t}\left(\hat{X}_{s} a^{\prime}\left(\hat{X}_{s}\right)-\check{X}_{s} a^{\prime}\left(\check{X}_{s}\right)\right) d s+\frac{1}{2} \int_{0}^{t}\left(a^{\prime \prime}\left(\hat{X}_{s}\right) \hat{\mu}_{s}(f)-a^{\prime \prime}\left(\check{X}_{s}\right) \check{\mu}_{s}(f)\right) \sigma^{2} d s \\
\quad+\int_{0}^{t}\left(a^{\prime}\left(\hat{X}_{s}\right) \sqrt{\hat{\mu}_{s}(f)}-a^{\prime}\left(\check{X}_{s}\right) \sqrt{\check{\mu}_{s}(f)}\right) \sigma d W_{s} \\
\quad-\int_{[0, t] \times \mathbb{R}_{+} \times \mathbb{R}}\left[a\left(\hat{X}_{s-}\right)-a\left(\check{X}_{s-}\right)\right] \mathbb{1}_{\left\{z \leq f\left(\hat{X}_{s-}\right) \wedge f\left(\check{X}_{s-}\right)\right\}} \pi(d s, d z, d u) \\
\quad+\int_{[0, t] \times \mathbb{R}_{+} \times \mathbb{R}}\left[a(0)-a\left(\hat{X}_{s-}\right)\right] \mathbb{1}_{\left\{f\left(\check{X}_{s-}\right)<z \leq f\left(\hat{X}_{s-}\right)\right\}} \pi(d s, d z, d u) \\
+\int_{[0, t] \times \mathbb{R}_{+} \times \mathbb{R}}\left[a\left(\check{X}_{s-}\right)-a(0)\right] \mathbb{1}_{\left\{f\left(\hat{X}_{s-}\right)<z \leq f\left(\check{X}_{s-}\right)\right\}} \pi(d s, d z, d u)=: A_{t}+M_{t}+\Delta_{t},
\end{gathered}
$$

where $A_{t}$ denotes the bounded variation part of the evolution, $M_{t}$ the martingale part and $\Delta_{t}$ the sum of the three jump terms. Notice that

$$
M_{t}=\int_{0}^{t}\left(a^{\prime}\left(\hat{X}_{s}\right) \sqrt{\hat{\mu}_{s}(f)}-a^{\prime}\left(\check{X}_{s}\right) \sqrt{\check{\mu}_{s}(f)}\right) \sigma d W_{s}
$$

is a square integrable martingale since $f$ and $a^{\prime}$ are bounded by Assumption 2.3.

We wish to obtain a control on $\left|Z_{t}^{*}\right|:=\sup _{s \leq t}\left|Z_{s}\right|$. We first take care of the jumps of $\left|Z_{t}\right|$. Notice first that, since $f$ and $a$ are bounded,

$$
\begin{aligned}
\Delta(x, y):=(f(x) \wedge f(y))|a(x)-a(y)|+|f(x)-f(y)||| a(0)-a(y) \mid & +|a(0)-a(x)| \mid \\
& \leq C|a(x)-a(y)|,
\end{aligned}
$$

implying that

$$
\mathbb{E} \sup _{s \leq t}\left|\Delta_{s}\right| \leq C \mathbb{E} \int_{0}^{t}\left|a\left(\hat{X}_{s}\right)-a\left(\check{X}_{s}\right)\right| d s \leq C t \mathbb{E}\left|Z_{t}^{*}\right| .
$$

Moreover, for a constant $C$ depending on $\sigma^{2},\|f\|_{\infty},\|a\|_{\infty},\left\|a^{\prime}\right\|_{\infty},\left\|a^{\prime \prime}\right\|_{\infty}$ and $\alpha$,

$$
\begin{aligned}
\mathbb{E} \sup _{s \leq t}\left|A_{s}\right| \leq C \int_{0}^{t} \mathbb{E} \mid a^{\prime}\left(\hat{X}_{s}\right) \hat{X}_{s} & -a^{\prime}\left(\check{X}_{s}\right) \check{X}_{s} \mid d s \\
& +C\left[\int_{0}^{t}\left|a^{\prime \prime}\left(\hat{X}_{s}\right)-a^{\prime \prime}\left(\check{X}_{s}\right)\right| d s+\int_{0}^{t}\left|\hat{\mu}_{s}(f)-\check{\mu}_{s}(f)\right| d s\right] .
\end{aligned}
$$

We know that

$$
\left|a^{\prime}\left(\hat{X}_{s}\right) \hat{X}_{s}-a^{\prime}\left(\check{X}_{s}\right) \check{X}_{s}\right|+\left|a^{\prime \prime}\left(\hat{X}_{s}\right)-a^{\prime \prime}\left(\check{X}_{s}\right)\right| \leq C\left|a\left(\hat{X}_{s}\right)-a\left(\check{X}_{s}\right)\right|=C\left|Z_{s}\right| .
$$

Therefore,

$$
\mathbb{E} \sup _{s \leq t}\left|A_{s}\right| \leq C \mathbb{E}\left[\int_{0}^{t}\left|Z_{s}\right| d s+\int_{0}^{t}\left|\hat{\mu}_{s}(f)-\check{\mu}_{s}(f)\right| d s\right] .
$$

Moreover,

$$
\left|\hat{\mu}_{s}(f)-\check{\mu}_{s}(f)\right|=\left|\mathbb{E}\left(f\left(\hat{X}_{s}\right)-f\left(\check{X}_{s}\right) \mid \mathcal{W}\right)\right| \leq \mathbb{E}\left(\left|f\left(\hat{X}_{s}\right)-f\left(\check{X}_{s}\right)\right| \mid \mathcal{W}\right) \leq \mathbb{E}\left(\left|Z_{s}\right| \mid \mathcal{W}\right),
$$

and thus,

$$
\mathbb{E} \int_{0}^{t}\left|\hat{\mu}_{s}(f)-\check{\mu}_{s}(f)\right| d s \leq \mathbb{E} \int_{0}^{t}\left|Z_{s}\right| d s \leq t \mathbb{E}\left|Z_{t}^{*}\right| .
$$


Putting all these upper bounds together we conclude that for a constant $C$ not depending on $t$,

$$
\mathbb{E} \sup _{s \leq t}\left|A_{s}\right| \leq C t \mathbb{E}\left|Z_{t}^{*}\right|
$$

Finally, we treat the martingale part using the Burkholder-Davis-Gundy inequality, and we obtain

$$
\mathbb{E} \sup _{s \leq t}\left|M_{s}\right| \leq C \mathbb{E}\left[\left(\int_{0}^{t}\left(a^{\prime}\left(\hat{X}_{s}\right) \sqrt{\hat{\mu}_{s}(f)}-a^{\prime}\left(\check{X}_{s}\right) \sqrt{\check{\mu}_{s}(f)}\right)^{2} d s\right)^{1 / 2}\right] .
$$

But

$$
\begin{gathered}
\left(a^{\prime}\left(\hat{X}_{s}\right) \sqrt{\hat{\mu}_{s}(f)}-a^{\prime}\left(\check{X}_{s}\right) \sqrt{\check{\mu}_{s}(f)}\right)^{2} \leq C\left[\left(\left(a^{\prime}\left(\hat{X}_{s}\right)-a^{\prime}\left(\check{X}_{s}\right)\right)^{2}+\left(\sqrt{\hat{\mu}_{s}(f)}-\sqrt{\check{\mu}_{s}(f)}\right)^{2}\right]\right. \\
\leq C\left|Z_{t}^{*}\right|^{2}+C\left(\sqrt{\hat{\mu}_{s}(f)}-\sqrt{\check{\mu}_{s}(f)}\right)^{2},
\end{gathered}
$$

where we have used that $\left|a^{\prime}(x)-a^{\prime}(y)\right| \leq C|a(x)-a(y)|$ and that $f$ and $a^{\prime}$ are bounded.

Finally, since inf $f>0$, such that $\sqrt{ }^{\cdot}$ is Lipschitz on [inf $f, \infty[$,

$$
\left|\sqrt{\hat{\mu}_{s}(f)}-\sqrt{\check{\mu}_{s}(f)}\right|^{2} \leq C\left|\hat{\mu}_{s}(f)-\check{\mu}_{s}(f)\right|^{2} \leq C\left(\mathbb{E}\left(\left|Z_{s}^{*}\right| \mid \mathcal{W}_{s}\right)\right)^{2} .
$$

We use that $\left|Z_{s}^{*}\right| \leq\left|Z_{t}^{*}\right|$, implying that $\mathbb{E}\left(\left|Z_{s}^{*}\right| \mid \mathcal{W}\right) \leq \mathbb{E}\left(\left|Z_{t}^{*}\right| \mid \mathcal{W}\right)$. Therefore we obtain the upper bound

$$
\left|\sqrt{\hat{\mu}_{s}(f)}-\sqrt{\check{\mu}_{s}(f)}\right|^{2} \leq C\left(\mathbb{E}\left(\left|Z_{t}^{*}\right| \mid \mathcal{W}\right)\right)^{2}
$$

for all $s \leq t$, which implies the control of

$$
\mathbb{E} \sup _{s \leq t}\left|M_{s}\right| \leq C \sqrt{t} \mathbb{E}\left|Z_{t}^{*}\right| .
$$

The above upper bounds imply that, for a constant $C$ not depending on $t$ nor on the initial condition,

$$
\mathbb{E}\left|Z_{t}^{*}\right| \leq C(t+\sqrt{t}) \mathbb{E}\left|Z_{t}^{*}\right|
$$

and therefore, for $t_{1}$ sufficiently small, $\mathbb{E}\left|Z_{t_{1}}^{*}\right|=0$. We can repeat this argument on intervals $\left[t_{1}, 2 t_{1}\right]$, with initial condition $\hat{X}_{t_{1}}$, and iterate it up to any finite $T$ because $t_{1}$ does only depend on the coefficients of the system but not on the initial condition. Recalling the definition of $\left|Z_{t}^{*}\right|$ and the fact that the function $a$ is increasing (and hence bijective), this implies the assertion.

Remark 2.8. Theorem 2.6 states the well-posedness of the SDE (2.4). Under the same hypotheses, with almost the same reasoning, one can prove the well-posedness of the system (2.2).

In the sequel, we shall also use an important property of the limit system (2.2), which is the conditional independence of the processes $\bar{X}^{i}(i \geq 1)$ given the Brownian motion $W$.

Proposition 2.9. If Assumptions 2.3 holds and $\int_{\mathbb{R}} x^{2} d \nu_{0}(x)<\infty$, then

(i) for all $N \in \mathbb{N}^{*}$ there exists a strong solution $\left(\bar{X}^{i}\right)_{1 \leq i \leq N}$ of (2.2), and pathwise uniqueness holds,

(ii) $\bar{X}^{1}, \ldots, \bar{X}^{N}$ are i.i.d. conditionally to $\mathcal{W}$,

(iii) for all $t \geq 0$, a.s., $\frac{1}{N} \sum_{i=1}^{N} \delta_{\bar{X}_{[0, t]}^{i}}$ converges weakly to $\lim _{N \rightarrow \infty} \frac{1}{N} \sum_{i=1}^{N} \delta_{\bar{X}_{[[0, t]}^{i}}=$ $P\left(\bar{X}_{\mid[0, t]}^{i} \in \cdot \mid \mathcal{W}_{t}\right)=P\left(\bar{X}_{\mid[0, t]}^{i} \in \cdot \mid \mathcal{W}\right)$. 
The proof of Proposition 2.9 is postponed to Section 4. Let us finally mention that the random limit measure $\mu$ satisfies a nonlinear stochastic PDE in weak form. More precisely,

Remark 2.10. Grant Assumption 2.3. Then the measure $\left.\left(\mu_{t}\right)_{t \geq 0}=\left(P\left(\bar{X}_{t}\right) \in \cdot \mid \mathcal{W}\right)\right)_{t \geq 0}$ satisfies the following nonlinear stochastic PDE in weak form: for any $\varphi \in C_{b}^{2}(\mathbb{R})$, for any $t \geq 0$,

$$
\begin{aligned}
\int_{\mathbb{R}} \varphi(x) \mu_{t}(d x)= & \int_{\mathbb{R}} \varphi(x) \nu_{0}(d x)+\int_{0}^{t}\left(\int_{\mathbb{R}} \varphi^{\prime}(x) \mu_{s}(d x)\right) \sqrt{\mu_{s}(f)} \sigma d W_{s} \\
& +\int_{0}^{t} \int_{\mathbb{R}}\left([\varphi(0)-\varphi(x)] f(x)-\alpha \varphi^{\prime}(x) x+\frac{1}{2} \sigma^{2} \varphi^{\prime \prime}(x) \mu_{s}(f)\right) \mu_{s}(d x) d s .
\end{aligned}
$$

\subsection{Another exchangeable system}

We have already stated the existence of a unique strong solution $\left(\bar{X}^{i}\right)_{i \geq 1}$ of the system (2.2). In the sequel we also need to show the well-posedness of the following exchangeable system of SDEs:

$$
\left\{\begin{aligned}
\bar{Y}_{t}^{i}= & \bar{Y}_{0}^{i}-\alpha \int_{0}^{t} \bar{Y}_{s}^{i} d s-\int_{[0, t] \times \mathbb{R}_{+}} \bar{Y}_{s-}^{i} \mathbb{1}_{\left\{z \leq f\left(\bar{Y}_{s-}^{i}\right)\right\}} \pi^{i}(d s, d z) \\
& +\sigma \int_{0}^{t} \sqrt{\mu_{t}(f)} d W_{s}, \\
& \text { are i.i.d., distributed } \sim \nu_{0},
\end{aligned}\right.
$$

where $\mu$ is the directing measure of the exchangeable system $\left(\bar{Y}^{i}\right)_{i \geq 1}{ }^{1}$ and $\mu_{t}$ its projection onto the $t$-th time coordinate. According to our previous reasoning, any solution of (2.2) is also solution of (2.7). But the converse is not obvious, because it is not a priori clear whether the Brownian motion is the only common noise of the system (2.7). We claim it in the next result.

Theorem 2.11. Grant Assumption 2.3 and suppose that $\int_{\mathbb{R}} x^{2} d \nu_{0}(x)<\infty$. Then there exists a unique strong solution $\left(\bar{Y}^{i}\right)_{i \geq 1}$ of (2.7). This solution is given by the unique strong solution of (2.2).

The proof of Theorem 2.11 is given in Section 4 .

\subsection{Convergence to the limit system}

In order to prove the convergence of the finite particle system to the limit system, we need to assume that the measure $\nu$ has a finite third moment.

\section{Assumption 2.12.}

$$
\int_{\mathbb{R}}|u|^{3} d \nu(u)<\infty .
$$

We are now able to state our main result.

Theorem 2.13. Grant Assumptions 2.2, 2.3 and 2.12. Then the empirical measure $\mu^{N}=\frac{1}{N} \sum_{i=1}^{N} \delta_{X^{N, i}}$ of the $N$-particle system $\left(X^{N, i}\right)_{1 \leq i \leq N}$ converges in distribution in $\mathcal{P}\left(D\left(\mathbb{R}_{+}, \mathbb{R}\right)\right)$ to $\mu:=\mathcal{L}\left(\bar{X}^{1} \mid \mathcal{W}\right)$, where $\left(\bar{X}^{i}\right)_{i \geq 1}$ is solution of $(2.2)$.

Corollary 2.14. Under the assumptions of Theorem $2.13,\left(X^{N, j}\right)_{1 \leq j \leq N}$ converges in distribution to $\left(\bar{X}^{j}\right)_{j \geq 1}$ in $D\left(\mathbb{R}_{+}, \mathbb{R}\right)^{\mathbb{N}^{*}}$, where $D\left(\mathbb{R}_{+}, \mathbb{R}\right)^{\mathbb{N}^{*}}$ is endowed with the product topology.

Proof. Together with the statement of Theorem 2.13, the proof is an immediate consequence of Proposition 7.20 of [1].

\footnotetext{
${ }^{1}$ that is, the $\left(\bar{Y}^{i}\right)_{i \geq 1}$ are i.i.d. of law $\mu$, conditionally on $\mu$
} 
Remark 2.15. In the statement of Corollary 2.14, we implicitly define $X^{N, i}:=0$ if $i>N$.

The following figure presents two simulations of the process $X^{N, 1}$ with $N=10$ and $N=1000$. Recalling that $X^{N, 1}$ is interpreted as the membrane potential of a given neuron in a network of $N$ neurons, we can see on the simulations the spiking times of this neuron, which are the times where the potential jumps to zero.
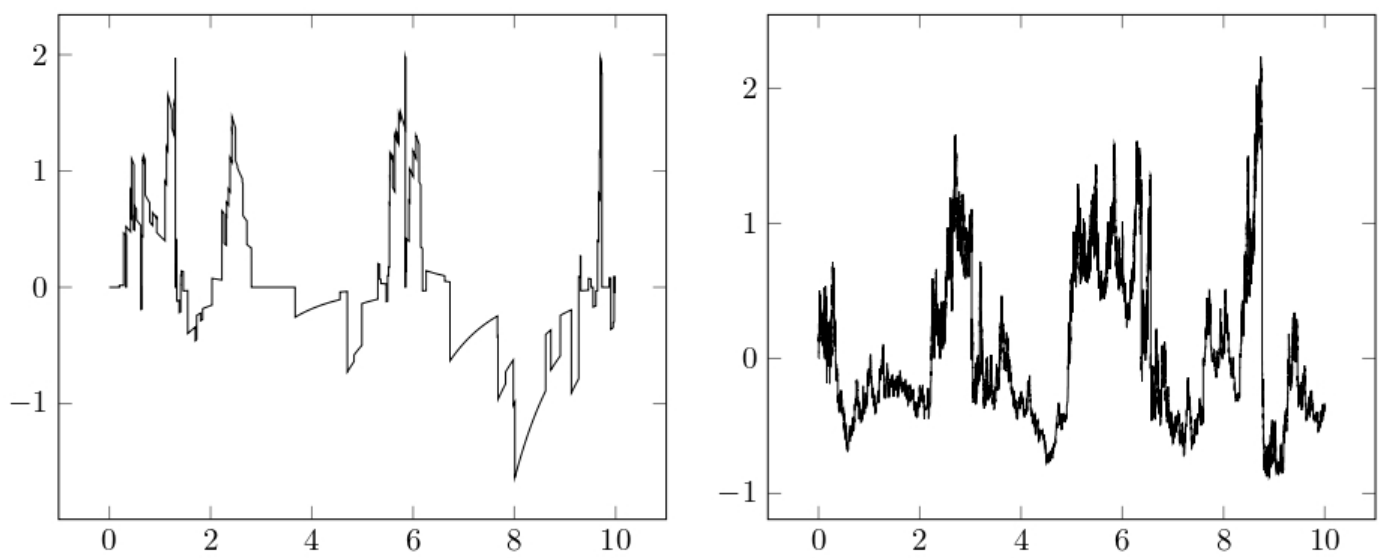

Figure 1: Simulation of trajectories of $\left(X_{t}^{N, 1}\right)_{0 \leq t \leq 10}$ with $X_{0}^{N, i}=0(1 \leq i \leq N), \alpha=1$, $\nu=\mathcal{N}(0,1), f(x)=2.2+1.4 \arctan (10 x-2), N=10$ (left picture) and $N=1000$ (right picture).

\section{Proof of Theorem 2.13}

This section is dedicated to prove that the sequence $\left(\mu^{N}\right)_{N}$ of the empirical measures $\mu^{N}:=\sum_{j=1}^{N} \delta_{\left(X_{t}^{N, j}\right)_{t>0}}$ converges in distribution to $\mu=\mathcal{L}\left(\bar{X}^{1} \mid \mathcal{W}\right)$, where $\left(\bar{X}^{j}\right)_{j \geq 1}$ is solution of (2.2).

In a first time, we prove that the sequence $\left(\mu^{N}\right)_{N}$ is tight on $\mathcal{P}\left(D\left(\mathbb{R}_{+}, \mathbb{R}\right)\right)$. The main step to prove the convergence of $\left(\mu^{N}\right)_{N}$ is then to show that each converging subsequence converges to the same limit in distribution. For this purpose, we introduce a new martingale problem, and we show that every possible limit of $\mu^{N}$ is a solution of this martingale problem. The result then follows from the fact that this martingale problem possesses a unique solution which is proved using the exchangeability of the associated system of processes.

\subsection{Tightness of $\left(\mu^{N}\right)_{N}$}

Proposition 3.1. Grant Assumptions 2.1 and 2.2, and assume that $f$ is bounded. For each $N \geq 1$, consider the unique solution $\left(X_{t}^{N}\right)_{t \geq 0}$ to (2.1) starting from some i.i.d. $\nu_{0}$-distributed initial conditions $X_{0}^{N, i}$.

(i) The sequence of processes $\left(X_{t}^{N, 1}\right)_{t \geq 0}$ is tight in $D\left(\mathbb{R}_{+}, \mathbb{R}\right)$.

(ii) The sequence of measures $\mu^{N}=N^{-1} \sum_{i=1}^{N} \delta_{\left(X_{t}^{N, i}\right)_{t \geq 0}}$ is tight in $\mathcal{P}\left(D\left(\mathbb{R}_{+}, \mathbb{R}\right)\right)$.

Proof. First, it is well-known that point (ii) follows from point (i) and the exchangeability of the system, see [16, Proposition 2.2-(ii)]. We thus only prove (i). To show that the family $\left(\left(X_{t}^{N, 1}\right)_{t \geq 0}\right)_{N \geq 1}$ is tight in $D\left(\mathbb{R}_{+}, \mathbb{R}\right)$, we use the criterion of Aldous, see Theorem VI.4.5 of [15]. It is sufficient to prove that 
(a) for all $T>0$, all $\varepsilon>0, \lim _{\delta \downarrow 0} \lim \sup _{N \rightarrow \infty} \sup _{\left(S, S^{\prime}\right) \in A_{\delta, T}} P\left(\left|X_{S^{\prime}}^{N, 1}-X_{S}^{N, 1}\right|>\varepsilon\right)=0$, where $A_{\delta, T}$ is the set of all pairs of stopping times $\left(S, S^{\prime}\right)$ such that $0 \leq S \leq S^{\prime} \leq$ $S+\delta \leq T$ a.s.,

(b) for all $T>0, \lim _{K \uparrow \infty} \sup _{N} P\left(\sup _{t \in[0, T]}\left|X_{t}^{N, 1}\right| \geq K\right)=0$.

To check (a), consider $\left(S, S^{\prime}\right) \in A_{\delta, T}$ and write

$$
\begin{aligned}
X_{S^{\prime}}^{N, 1}-X_{S}^{N, 1}=-\int_{S}^{S^{\prime}} \int_{\mathbb{R}} \int_{0}^{\infty} X_{s-}^{N, 1} & \mathbb{1}_{\left\{z \leq f\left(X_{s-}^{N, 1}\right)\right\}} \pi^{1}(d s, d u, d z)-\alpha \int_{S}^{S^{\prime}} X_{s}^{N, 1} d s \\
& +\frac{1}{\sqrt{N}} \sum_{j=2}^{N} \int_{S}^{S^{\prime}} \int_{\mathbb{R}} \int_{0}^{\infty} u \mathbb{1}_{\left\{z \leq f\left(X_{s-}^{N, j}\right)\right\}} \pi^{j}(d s, d u, d z),
\end{aligned}
$$

implying that

$$
\begin{aligned}
& \left|X_{S^{\prime}}^{N, 1}-X_{S}^{N, 1}\right| \leq\left|\int_{S} \int_{\mathbb{R}}^{S^{\prime}} \int_{0}^{\infty} X_{s-}^{N, 1} \mathbb{1}_{\left\{z \leq f\left(X_{s-}^{N, 1}\right)\right\}} \pi^{1}(d s, d u, d z)\right| \\
& \quad+\delta \alpha \sup _{0 \leq s \leq T}\left|X_{s}^{N, 1}\right|+\left|\frac{1}{\sqrt{N}} \sum_{j=2}^{N} \int_{S}^{S^{\prime}} \int_{\mathbb{R}} \int_{0}^{\infty} u \mathbb{1}_{\left\{z \leq f\left(X_{s-}^{N, j}\right)\right\}} \pi^{j}(d s, d u, d z)\right| \\
& =:\left|I_{S, S^{\prime}}\right|+\delta \alpha \sup _{0 \leq s \leq T}\left|X_{s}^{N, 1}\right|+\left|J_{S, S^{\prime}}\right| .
\end{aligned}
$$

Note that $\left|I_{S, S^{\prime}}\right|>0$ implies that $\tilde{I}_{S, S^{\prime}}:=\int_{S}^{S^{\prime}} \int_{\mathbb{R}} \int_{0}^{\infty} \mathbb{1}_{\left\{z \leq f\left(X_{s-}^{N, 1}\right)\right\}} \pi^{i}(d s, d u, d z) \geq 1$, and thus

$$
P\left(\left|I_{S, S^{\prime}}\right|>0\right) \leq P\left(\tilde{I}_{S, S^{\prime}} \geq 1\right) \leq \mathbb{E}\left[\tilde{I}_{S, S^{\prime}}\right] \leq \mathbb{E}\left[\int_{S}^{S+\delta} f\left(X_{s}^{N, 1}\right) d s\right] \leq\|f\|_{\infty} \delta,
$$

since $f$ is bounded. We proceed similarly to check that

$$
P\left(\left|J_{S, S^{\prime}}\right| \geq \varepsilon\right) \leq \frac{1}{\varepsilon^{2}} \mathbb{E}\left[\left(J_{S, S^{\prime}}\right)^{2}\right] \leq \frac{\sigma^{2}}{N \varepsilon^{2}} \sum_{j=2}^{N} \mathbb{E}\left[\int_{S}^{S+\delta} f\left(X_{s}^{N, j}\right) d s\right] \leq \frac{\sigma^{2}}{\varepsilon^{2}}\|f\|_{\infty} \delta .
$$

The term $\sup _{0 \leq s \leq T}\left|X_{s}^{N, 1}\right|$ can be handled using Lemma 4.1.(ii).

Finally (b) is a straightforward consequence of Lemma 4.1.(ii) and Markov's inequality.

\subsection{Martingale problem}

We now introduce a new martingale problem, whose solutions are the limits of any converging subsequence of $\mu^{N}=\frac{1}{N} \sum_{j=1}^{N} \delta_{X^{N, j}}$. In this martingale problem, we are interested in couples of trajectories to be able to put hands on the correlations between the particles. In particular, this will allow us to show that, in the limit system (2.2), the processes $\bar{X}^{i}(i \geq 1)$ share the same Brownian motion, but are driven by Poisson measures $\pi^{i}(i \geq 1)$ which are independent. The reason why we only need to study the correlation between two particles is the exchangeability of the infinite system.

Let $Q$ be a probability measure on $\mathcal{P}\left(D\left(\mathbb{R}_{+}, \mathbb{R}\right)\right)$. Define a probability measure $P$ on $\mathcal{P}\left(D\left(\mathbb{R}_{+}, \mathbb{R}\right)\right) \times D\left(\mathbb{R}_{+}, \mathbb{R}\right)^{2}$ by

$$
P(A \times B):=\int_{\mathcal{P}\left(D\left(\mathbb{R}_{+}, \mathbb{R}\right)\right)} \mathbb{1}_{A}(m) m \otimes m(B) Q(d m) .
$$

We write any atomic event $\omega \in \Omega:=\mathcal{P}\left(D\left(\mathbb{R}_{+}, \mathbb{R}\right)\right) \times D\left(\mathbb{R}_{+}, \mathbb{R}\right)^{2}$ as $\omega=(\mu, Y)$, with $Y=\left(Y^{1}, Y^{2}\right)$. Thus, the law of the canonical variable $\mu$ is $Q$, and, conditionally on $\mu$, the law of $\left(Y^{1}, Y^{2}\right)$ is $\mu \otimes \mu$. 
Writing $\mu_{t}:=\int_{D\left(\mathbb{R}_{+}, \mathbb{R}\right)} \mu(d \gamma) \delta_{\gamma_{t}}$ for the projection onto the $t$-th time coordinate, we introduce the filtration

$$
\mathcal{G}_{t}=\sigma\left(Y_{s}, s \leq t\right) \vee \sigma\left(\mu_{s}(f), s \leq t\right) .
$$

Definition 3.2. We say that $Q \in \mathcal{P}\left(\mathcal{P}\left(D\left(\mathbb{R}_{+}, \mathbb{R}\right)\right)\right)$ is a solution to the martingale problem $(\mathcal{M})$ if the following holds.

(i) $Q$-almost surely, $\mu_{0}=\nu_{0}$.

(ii) For all $g \in C_{b}^{2}\left(\mathbb{R}^{2}\right), M_{t}^{g}:=g\left(Y_{t}\right)-g\left(Y_{0}\right)-\int_{0}^{t} L g\left(\mu_{s}, Y_{s}\right) d s$ is a $\left(P,\left(\mathcal{G}_{t}\right)_{t}\right)$-martingale, where

$$
\begin{aligned}
L g(\mu, x)= & -\alpha x^{1} \partial_{x^{1}} g(x)-\alpha x^{2} \partial_{x^{2}} g(x)+\frac{\sigma^{2}}{2} \mu(f) \sum_{i, j=1}^{2} \partial_{x^{i} x^{j}}^{2} g(x) \\
& +f\left(x^{1}\right)\left(g\left(0, x^{2}\right)-g(x)\right)+f\left(x^{2}\right)\left(g\left(x^{1}, 0\right)-g(x)\right) .
\end{aligned}
$$

Let $\left(\bar{X}^{i}\right)_{i \geq 1}$ be the solution of the limit system (2.2) and $\mu=\mathcal{L}\left(\bar{X}^{1} \mid \mathcal{W}\right)$. Then Proposition 2.9.(ii) and Lemma (2.12).(a) of [1] imply that $\mu$ is the directing measure of $\left(\bar{X}^{i}\right)_{i \geq 1}$. Thus the law of $\left(\mu, \bar{X}^{1}, \bar{X}^{2}\right)$ is $P$ given in (3.1). And, by Itô's formula, $\left(\bar{X}^{1}, \bar{X}^{2}\right)$ satisfies the martingale property of Definition 3.2. In other words, $\mathcal{L}(\mu)$ is a solution of $(\mathcal{M})$.

Let us now characterize any possible solution of $(\mathcal{M})$, which is the first step to prove uniqueness of the solution of $(\mathcal{M})$.

Lemma 3.3. Let $Q \in \mathcal{P}\left(\mathcal{P}\left(D\left(\mathbb{R}_{+}, \mathbb{R}\right)\right)\right)$. Assume that $Q$ is a solution of $(\mathcal{M})$ and that $f$ is bounded. Let $(\mu, Y)$ be the canonical variable defined above, and write $Y=\left(Y^{1}, Y^{2}\right)$. Then there exists a standard $\left(\mathcal{G}_{t}\right)_{t}-$ Brownian motion $W$ and on an extension $\left(\tilde{\Omega},\left(\tilde{\mathcal{G}}_{t}\right)_{t}, \tilde{P}\right)$ of $\left(\Omega,\left(\mathcal{G}_{t}\right)_{t}, P\right)$ there exist $\left(\tilde{\mathcal{G}}_{t}\right)_{t}-$ Poisson random measures $\pi^{1}, \pi^{2}$ on $\mathbb{R}_{+} \times \mathbb{R}_{+}$having Lebesgue intensity such that $W, \pi^{1}$ and $\pi^{2}$ are independent and

$$
\begin{aligned}
& d Y_{t}^{1}=-\alpha Y_{t}^{1} d t+\sigma \sqrt{\mu_{t}(f)} d W_{t}-Y_{t-}^{1} \int_{\mathbb{R}_{+}} \mathbb{1}_{\left\{z \leq f\left(Y_{t-}^{1}\right)\right\}} \pi^{1}(d t, d z), \\
& d Y_{t}^{2}=-\alpha Y_{t}^{2} d t+\sigma \sqrt{\mu_{t}(f)} d W_{t}-Y_{t-}^{2} \int_{\mathbb{R}_{+}} \mathbb{1}_{\left\{z \leq f\left(Y_{t-}^{2}\right)\right\}} \pi^{2}(d t, d z) .
\end{aligned}
$$

Proof. Item (ii) of $(\mathcal{M})$ together with Theorem II.2.42 of [15] imply that $Y$ is a semimartingale with characteristics $(B, C, \nu)$ given by

$$
\begin{aligned}
& B_{t}^{i}=-\alpha \int_{0}^{t} Y_{s}^{i} d s-\int_{0}^{t} Y_{s}^{i} f\left(Y_{s}^{i}\right) d s, \quad 1 \leq i \leq 2, \\
& C_{t}^{i, j}=\int_{0}^{t} \mu_{s}(f) d s, \quad 1 \leq i, j \leq 2, \\
& \nu(d t, d y)=d t\left(f\left(Y_{t-}^{1}\right) \delta_{\left(-Y_{t-}^{1}, 0\right)}(d y)+f\left(Y_{t-}^{2}\right) \delta_{\left(0,-Y_{t-}^{2}\right)}(d y)\right) .
\end{aligned}
$$

Then we can use the canonical representation of $Y$ (see Theorem II.2.34 of [15]) with the truncation function $h(y)=y$ for every $y: Y_{t}-Y_{0}-B_{t}=M_{t}^{c}+M_{t}^{d}$, where $M^{c}$ is a continuous local martingale and $M^{d}$ a purely discontinuous local martingale. By definition of the characteristics, $\left\langle M^{c, i}, M^{c, j}\right\rangle_{t}=C_{t}^{i, j}$. In particular, $\left\langle M^{c, i}\right\rangle_{t}=\int_{0}^{t} \mu_{s}(f) d s$ $(i=1,2)$. Consequently, applying Theorem II.7.1' of [14] to the 2-dimensional martingale $\left(M^{c, 1}, M^{c, 2}\right)$, we know that there exists a Brownian motion $W$ such that

$$
M_{t}^{c, i}=\int_{0}^{t} \sqrt{\mu_{s}(f)} d W_{s}, \quad i=1,2 .
$$

We now prove the existence of the independent Poisson measures $\pi^{1}, \pi^{2}$. We know that $M^{d}=h *\left(\mu^{Y}-\nu\right)$, where $\mu^{Y}=\sum_{s} \mathbb{1}_{\left\{\Delta Y_{s} \neq 0\right\}} \delta_{\left(s, Y_{s}\right)}$ is the jump measure of $Y$ and $\nu$ 
is its compensator. We rely on Theorem II.7.4 of [14]. Using the notation therein, we introduce $Z=\mathbb{R}_{+}, m$ the Lebesgue measure on $Z$ and

$$
\theta(t, z):=\left(-Y_{t-}^{1}, 0\right) \mathbb{1}_{\left\{z \leq f\left(Y_{t-}^{1}\right)\right\}}+\left(0,-Y_{t-}^{2}\right) \mathbb{1}_{\left\{\|f\|_{\infty}<z \leq\|f\|_{\infty}+f\left(Y_{t-}^{2}\right)\right\}} .
$$

According to Theorem II.7.4 of [14], there exists a Poisson measure $\pi$ on $\mathbb{R}_{+} \times \mathbb{R}_{+}$having intensity $d t \cdot d z$ such that, for all $E \in \mathcal{B}\left(\mathbb{R}^{2}\right)$,

$$
\mu^{Y}([0, t] \times E)=\int_{0}^{t} \int_{0}^{\infty} \mathbb{1}_{\{\theta(s, z) \in E\}} \pi(d s, d z) .
$$

In what follows we show how to construct two independent Poisson random measures $\pi^{1}$ and $\pi^{2}$ from $\pi$ with the desired representation property, using two disjoint parts of $\pi$. For $\pi^{1}$ we use $\pi_{\mid \mathbb{R}_{+} \times\left[0,|| f \|_{\infty}\right]}$, and for $\pi^{2}$ we use $\pi_{\mid \mathbb{R}_{+} \times\left[\|f\|_{\infty}, 2\|f\|_{\infty}\right]}$ such that the Poisson measures $\pi^{1}$ and $\pi^{2}$ will be independent.

To construct $\pi_{1}$ and $\pi_{2}$, we also consider two independent Poisson measures $\tilde{\pi}^{1}, \tilde{\pi}^{2}$ (independent of everything else) on $\left[\|f\|_{\infty}, \infty[\right.$ having Lebesgue intensity. We then define $\pi^{1}$ in the following way: For any $A \in \mathcal{B}\left(\mathbb{R}_{+} \times\left[0,\|f\|_{\infty}\right]\right), \pi^{1}(A)=\pi(A)$, and for $A \in \mathcal{B}\left(\mathbb{R}_{+} \times\right]\|f\|_{\infty}, \infty[), \pi^{1}(A)=\tilde{\pi}^{1}(A)$. We define $\pi^{2}$ in a similar way: For $A \in$ $\mathcal{B}\left(\mathbb{R}_{+} \times\left[0,\|f\|_{\infty}\right]\right), \pi^{2}(A)=\pi\left(\left\{\left(t,\|f\|_{\infty}+z\right):(t, z) \in A\right\}\right)$, and for $A \in \mathcal{B}\left(\mathbb{R}_{+} \times\right]\|f\|_{\infty}, \infty[)$, $\pi^{2}(A)=\tilde{\pi}^{2}(A)$. By definition of Poisson random measures, $\pi^{1}$ and $\pi^{2}$ are independent Poisson measures on $\mathbb{R}_{+}^{2}$ having Lebesgue intensity, and together with (3.2), we have the desired representation

$$
M_{t}^{d, i}=-\int_{[0, t] \times \mathbb{R}_{+}} Y_{s-}^{i} \mathbb{1}_{\left\{z \leq f\left(Y_{s-}^{i}\right)\right\}} \pi^{i}(d s, d z)+\int_{0}^{t} Y_{s}^{i} f\left(Y_{s}^{i}\right) d s, \quad 1 \leq i \leq 2 .
$$

In the next step we prove that there exists at most one (and thus exactly one) solution for the martingale problem $(\mathcal{M})$ using Lemma 3.3 and Theorem 2.11.

Theorem 3.4. Grant Assumptions 2.2, 2.3 and 2.12. Then there is a unique solution $Q$ of the martingale problem $(\mathcal{M})$. This solution can be written as $Q=\mathcal{L}(\mu)$, with $\mu=\mathcal{L}(\bar{X} \mid \mathcal{W})$, where $\bar{X}$ is the unique strong solution of (2.4).

The main idea of the proof is to apply Lemma 3.3 to recover the system of SDEs (2.7) and then to rely on Theorem 2.11.

Proof. Let $Q \in \mathcal{P}\left(\mathcal{P}\left(D\left(\mathbb{R}_{+}, \mathbb{R}\right)\right)\right)$ be a solution of $(\mathcal{M})$ and write $Q=\mathcal{L}(\mu)$. The proof consists in showing that $\mu$ is the distribution of the directing measure of the system (2.7), which is unique by Theorem 2.11 .

To begin with, we can assume that $\mu$ is the directing measure of some exchangeable system $\left(\bar{Y}^{i}\right)_{i \geq 1}$. Indeed, it is sufficient to work on the canonical space

$$
\Omega^{\prime}=\mathcal{P}\left(D\left(\mathbb{R}_{+}, \mathbb{R}\right)\right) \times D\left(\mathbb{R}_{+}, \mathbb{R}\right)^{\mathbb{N}^{*}}
$$

endowed with the probability measure $P^{\prime}$ defined as follows. For all $A \in \mathcal{B}\left(\mathcal{P}\left(D\left(\mathbb{R}_{+}, \mathbb{R}\right)\right)\right)$ and $B_{k} \in \mathcal{B}\left(D\left(\mathbb{R}_{+}, \mathbb{R}\right)\right)(k \geq 1)$ where at most a finite number of sets $B_{k}(k \geq 1)$ are different from $D\left(\mathbb{R}_{+}, \mathbb{R}\right)$,

$$
P^{\prime}\left(A \times B_{1} \times \ldots \times B_{k} \times \ldots\right)=\int_{\mathcal{P}\left(D\left(\mathbb{R}_{+}, \mathbb{R}\right)\right)} \mathbb{1}_{A}(m) m^{\otimes \mathbb{N}^{*}}\left(B_{1} \times \ldots \times B_{k} \times \ldots\right) Q(d m) .
$$

Then, noting $\left(\mu, \bar{Y}^{1}, \bar{Y}^{2}, \ldots, \bar{Y}^{k}, \ldots\right)$ the canonical random variables on $\Omega^{\prime}$, we know that $\mu$ is the directing measure of the exchangeable system $\left(\bar{Y}^{i}\right)_{i \geq 1}$. In particular, for all $i \neq j$,

$$
\mathcal{L}\left(\mu,\left(\bar{Y}^{i}, \bar{Y}^{j}\right)\right)=P,
$$


where $P$ is given by (3.1), with $Q=\mathcal{L}(\mu)$.

Thanks to Lemma 3.3 we know that there exist Brownian motions $W^{(i, j)}(i, j \geq 1)$ and Poisson random measures $\pi^{(i, j), 1}, \pi^{(i, j), 2}(i, j \geq 1)$ such that for all pairs $(i, j), i \neq j$, $\pi^{(i, j), 1}$ is independent of $\pi^{(i, j), 2}$ and such that

$$
\begin{aligned}
& d \bar{Y}_{t}^{i}=-\alpha \bar{Y}_{t}^{i} d t+\sigma \sqrt{\mu_{t}(f)} d W_{t}^{(i, j)}-\bar{Y}_{t-}^{i} \int_{\mathbb{R}_{+}} \mathbb{1}_{\left\{z \leq f\left(\bar{Y}_{t-}^{i}\right)\right\}} \pi^{(i, j), 1}(d t, d z), \\
& d \bar{Y}_{t}^{j}=-\alpha \bar{Y}_{t}^{j} d t+\sigma \sqrt{\mu_{t}(f)} d W_{t}^{(i, j)}-\bar{Y}_{t-}^{j} \int_{\mathbb{R}_{+}} \mathbb{1}_{\left\{z \leq f\left(\bar{Y}_{t-}^{j}\right)\right\}} \pi^{(i, j), 2}(d t, d z) .
\end{aligned}
$$

The exchangeability of the system $\left(\bar{Y}^{i}\right)_{i \geq 1}$ implies that for all distinct $i, j \geq 1, j>1$, $\mathcal{L}\left(\bar{X}^{1}, \bar{X}^{2}\right)=\mathcal{L}\left(\bar{X}^{1}, \bar{X}^{j}\right)=\mathcal{L}\left(\bar{X}^{i}, \bar{X}^{j}\right)$. Thus $W:=W^{(1,2)}=W^{(1, j)}=W^{(i, j)}$ for all $i \neq j$. Besides, as for all distinct $i, j, k \geq 1, \mathcal{L}\left(\bar{X}^{i}, \bar{X}^{j}\right)=\mathcal{L}\left(\bar{X}^{i}, \bar{X}^{k}\right)=\mathcal{L}\left(\bar{X}^{j}, \bar{X}^{i}\right)$, we also know that $\pi^{i}:=\pi^{(i, j), 1}=\pi^{(i, k), 1}=\pi^{(j, i), 2}$. As a consequence, the Poisson measures $\pi^{i}$ $(i \geq 1)$ are pairwise independent, and so they are also mutually independent, since the independence between Poisson measures is characterized by the fact that their supports (i.e. the sets $\left.\left\{t \geq 0: \pi^{i}\left(\{t\} \times \mathbb{R}_{+}\right) \neq 0\right\}\right)$ are disjoint (see Theorem II.6.3 of [14]).

We summarize the above step. We have just shown that there exist a Brownian motion $W$ and independent Poisson random measures $\pi^{i}(i \geq 1)$ with Lebesgue intensity, independent of $W$, such that, for all $i \geq 1$,

$$
d \bar{Y}_{t}^{i}=-\alpha \bar{Y}_{t}^{i} d t+\sigma \sqrt{\mu_{t}(f)} d W_{t}-\bar{Y}_{t-}^{i} \int_{\mathbb{R}_{+}} \mathbb{1}_{\left\{z \leq f\left(\bar{Y}_{t-}^{i}\right)\right\}} \pi^{i}(d t, d z) .
$$

As a consequence, $\left(\bar{Y}^{i}\right)_{i \geq 1}$ is solution to (2.7), and Theorem 2.11 allows to conclude.

The last missing point to prove our main result, Theorem 2.13, is the following

Theorem 3.5. Assume that Assumptions 2.1, 2.2 and 2.12 hold, and that $f$ is bounded. Then the distribution of any limit $\mu$ of the sequence $\mu^{N}:=\frac{1}{N} \sum_{j=1}^{N} \delta_{X^{N, j}}$ is solution of item (ii) of $(\mathcal{M})$.

Proof. Step 1. We first check that for any $t \geq 0$, a.s., $\mu(\{\gamma: \Delta \gamma(t) \neq 0\})=0$, where $\Delta \gamma(t):=\gamma(t)-\gamma(t-)$. We assume by contradiction that there exists $t>0$ such that $\mu(\{\gamma: \Delta \gamma(t) \neq 0\})>0$ with positive probability. Hence there are $a, b>0$ such that the event $E:=\{\mu(\{\gamma:|\Delta \gamma(t)|>a\})>b\}$ has a positive probability. For every $\varepsilon>0$, we have $E \subset\left\{\mu\left(\mathcal{B}_{a}^{\varepsilon}\right)>b\right\}$, where $\mathcal{B}_{a}^{\varepsilon}:=\left\{\gamma: \sup _{s \in(t-\varepsilon, t+\varepsilon)}|\Delta \gamma(s)|>a\right\}$, which is an open subset of $D\left(\mathbb{R}_{+}, \mathbb{R}\right)$. Thus $\mathcal{P}_{a, b}^{\varepsilon}:=\left\{\mu \in \mathcal{P}\left(D\left(\mathbb{R}_{+}, \mathbb{R}\right)\right): \mu\left(\mathcal{B}_{a}^{\varepsilon}\right)>b\right\}$ is an open subset of $\mathcal{P}\left(D\left(\mathbb{R}_{+}, \mathbb{R}\right)\right)$. The Portmanteau theorem implies then that for any $\varepsilon>0$,

$$
\liminf _{N \rightarrow \infty} P\left(\mu^{N} \in \mathcal{P}_{a, b}^{\varepsilon}\right) \geq P\left(\mu \in \mathcal{P}_{a, b}^{\varepsilon}\right) \geq P(E)>0 .
$$

Firstly, we can write

$$
J^{N, \varepsilon, i}:=\sup _{t-\varepsilon<s<t+\varepsilon}\left|\Delta X_{s}^{N, i}\right|=G_{N}^{\varepsilon, i} \vee S_{N}^{\varepsilon}
$$

where $G_{N}^{\varepsilon, i}:=\max _{s \in D_{N}^{\varepsilon, i}}\left|X_{s-}^{N, i}\right|$ is the maximal height of the big jumps of $X^{N, i}$, with $D_{N}^{\varepsilon, i}:=$ $\left\{t-\varepsilon \leq s \leq t+\varepsilon: \pi^{i}\left(\{s\} \times\left[0, f\left(X_{s-}^{N, i}\right)\right] \times \mathbb{R}_{+}\right) \neq 0\right\}$. Moreover, $S_{N}^{\varepsilon}:=\max \left\{\left|U^{j}(s)\right| / \sqrt{N}: s \in\right.$ $\left.\bigcup_{1 \leq j \leq N} D_{N}^{\varepsilon, j}\right\}$ is the maximal height of the small jumps of $X^{N, i}$, where $U^{j}(s)$ is defined for $s \in D_{N}^{\varepsilon, j}$, almost surely, as the only real number that satisfies $\pi^{j}\left(\{s\} \times\left[0, f\left(X_{s-}^{N, j}\right)\right] \times\right.$ $\left.\left\{U^{j}(s)\right\}\right)=1$.

We have that

$$
\left\{\mu^{N}\left(\mathcal{B}_{a}^{\varepsilon}\right)>b\right\}=\left\{\frac{1}{N} \sum_{j=1}^{N} \mathbb{1}_{\left\{J^{N, \varepsilon, j}>a\right\}}>b\right\}
$$


Consequently, by exchangeability and Markov's inequality,

$P\left(\mu^{N}\left(\mathcal{B}_{a}^{\varepsilon}\right)>b\right) \leq \frac{1}{b} \mathbb{E}\left[\mathbb{1}_{\left\{J^{N, \varepsilon, 1}>a\right\}}\right]=\frac{1}{b} P\left(J^{N, \varepsilon, 1}>a\right) \leq \frac{1}{b}\left(P\left(G_{N}^{\varepsilon, 1}>a\right)+P\left(S_{N}^{\varepsilon}>a\right)\right)$.

The number of big jumps of $X^{N, 1}$ in $] t-\varepsilon, t+\varepsilon[$ is smaller than a random variable $\xi$ having Poisson distribution with parameter $2 \varepsilon\|f\|_{\infty}$. Hence

$$
P\left(G_{N, 1}^{\varepsilon}>a\right) \leq P(\xi \geq 1)=1-e^{2 \varepsilon\|f\|_{\infty}} \leq 2 \varepsilon\|f\|_{\infty} .
$$

The small jumps that occur in $] t-\varepsilon, t+\varepsilon\left[\right.$ are included in $\left\{U_{1} / \sqrt{N}, \ldots, U_{K} / \sqrt{N}\right\}$ where $K$ is a $\mathbb{N}$-valued random variable having Poisson distribution with parameter $2 \varepsilon N\|f\|_{\infty}$, which is independent of the variables $U_{i}(i \geq 1)$ that are i.i.d. with distribution $\nu$. Hence,

$$
P\left(S_{N}^{\varepsilon}>a\right) \leq P\left(\max _{1 \leq i \leq K} \frac{\left|U_{i}\right|}{\sqrt{N}}>a\right) \leq \mathbb{E}\left[\mathbb{P}\left(\max _{1 \leq i \leq K} \frac{\left|U_{i}\right|}{\sqrt{N}}>a \mid K\right)\right]=\mathbb{E}[\psi(K)],
$$

where $\psi(k)=P\left(\max _{1 \leq i \leq k}\left|U_{i}\right|>a \sqrt{N}\right) \leq k P\left(\left|U_{1}\right|>a \sqrt{N}\right) \leq k a^{-2} N^{-1} \mathbb{E}\left[U_{1}^{2}\right]$. Hence

$$
P\left(S_{N}^{\varepsilon}>a\right) \leq \frac{\mathbb{E}\left[U_{1}^{2}\right]}{N a^{2}} \mathbb{E}[K] \leq 2\|f\|_{\infty} \mathbb{E}\left[U_{1}^{2}\right] \frac{1}{a} \varepsilon .
$$

Inserting the bounds (3.5) and (3.6) in (3.4), we have

$$
P\left(\mu^{N}\left(\mathcal{B}_{a}^{\varepsilon}\right)>b\right) \leq C \varepsilon,
$$

where $C$ does not depend on $N$ nor $\varepsilon$. This last inequality is in contradiction with (3.3) since $P(E)$ does not depend on $\varepsilon$.

Step 2. In the following, we note $\partial^{2} \varphi:=\sum_{i, j=1}^{2} \partial_{x^{i} x^{j}}^{2} \varphi$. For any $0 \leq s_{1}<\ldots<s_{k}<s<t$, any $\psi_{1}, \ldots, \psi_{k} \in C_{b}(\mathbb{R}), \varphi_{1}, \ldots, \varphi_{k} \in C_{b}\left(\mathbb{R}^{2}\right)$ and any $\varphi \in C_{c}^{3}\left(\mathbb{R}^{2}\right)$, we introduce

$$
\begin{aligned}
& F(\mu):=\psi_{1}\left(\mu_{s_{1}}(f)\right) \ldots \psi_{k}\left(\mu_{s_{k}}(f)\right) \int_{D\left(\mathbb{R}_{+}, \mathbb{R}\right)^{2}} \mu \otimes \mu(d \gamma) \varphi_{1}\left(\gamma_{s_{1}}\right) \ldots \varphi_{k}\left(\gamma_{s_{k}}\right) \\
& {\left[\varphi\left(\gamma_{t}\right)-\varphi\left(\gamma_{s}\right)+\alpha \int_{s}^{t} \gamma_{r}^{1} \partial_{x^{1}} \varphi\left(\gamma_{r}\right) d r+\alpha \int_{s}^{t} \gamma_{r}^{2} \partial_{x^{2}} \varphi\left(\gamma_{r}\right) d r-\frac{\sigma^{2}}{2} \int_{s}^{t} \mu_{r}(f) \partial^{2} \varphi\left(\gamma_{r}\right) d r\right.} \\
& \left.\quad-\int_{s}^{t} f\left(\gamma_{r}^{1}\right)\left(\varphi\left(0, \gamma_{r}^{2}\right)-\varphi\left(\gamma_{r}\right)\right) d r-\int_{s}^{t} f\left(\gamma_{r}^{2}\right)\left(\varphi\left(\gamma_{r}^{1}, 0\right)-\varphi\left(\gamma_{r}\right)\right) d r\right] .
\end{aligned}
$$

Let us note that $F$ is bounded, because we have chosen a compactly supported test function $\varphi$. To show that $\mathcal{L}(\mu)$ is solution of item (ii) of the martingale problem $(\mathcal{M})$, by a classical density argument, it is sufficient to prove that $\mathbb{E}[F(\mu)]=0$. We have

$$
\begin{gathered}
F\left(\mu^{N}\right)=\psi_{1}\left(\mu_{s_{1}}^{N}(f)\right) \ldots \psi_{k}\left(\mu_{s_{k}}^{N}(f)\right) \frac{1}{N^{2}} \sum_{i=1}^{N} \sum_{j=1}^{N} \varphi_{1}\left(X_{s_{1}}^{N, i}, X_{s_{1}}^{N, j}\right) \ldots \varphi_{k}\left(X_{s_{k}}^{N, i}, X_{s_{k}}^{N, j}\right) . \\
{\left[\varphi\left(X_{t}^{N, i}, X_{t}^{N, j}\right)-\varphi\left(X_{s}^{N, i}, X_{s}^{N, j}\right)+\alpha \int_{s}^{t} X_{r}^{N, i} \partial_{x^{1}} \varphi\left(X_{r}^{N, i}, X_{r}^{N, j}\right) d r\right.} \\
+\alpha \int_{s}^{t} X_{r}^{N, j} \partial_{x^{2}} \varphi\left(X_{r}^{N, i}, X_{r}^{N, j}\right) d r-\frac{\sigma^{2}}{2} \int_{s}^{t} \mu_{r}^{N}(f) \partial^{2} \varphi\left(X_{r}^{N, i}, X_{r}^{N, j}\right) d r \\
-\int_{s}^{t} f\left(X_{r}^{N, i}\right)\left(\varphi\left(0, X_{r}^{N, j}\right)-\varphi\left(X_{r}^{N, i}, X_{r}^{N, j}\right)\right) d r \\
\left.-\int_{s}^{t} f\left(X_{r}^{N, j}\right)\left(\varphi\left(X_{r}^{N, i}, 0\right)-\varphi\left(X_{r}^{N, i}, X_{r}^{N, j}\right)\right) d r\right] .
\end{gathered}
$$




\section{Conditional propagation of chaos}

But recalling (2.1) and using Itô's formula, for any $i \neq j$, we have

$$
\begin{aligned}
& \varphi\left(X_{t}^{N, i}, X_{t}^{N, j}\right) \\
& =\varphi\left(X_{s}^{N, i}, X_{s}^{N, j}\right)-\alpha \int_{s}^{t} X_{r}^{N, i} \partial_{x^{1}} \varphi\left(X_{r}^{N, i}, X_{r}^{N, j}\right) d r-\alpha \int_{s}^{t} X_{r}^{N, j} \partial_{x^{2}} \varphi\left(X_{r}^{N, i}, X_{r}^{N, j}\right) d r \\
& +\int_{] s, t] \times \mathbb{R}_{+} \times \mathbb{R}} \mathbb{1}_{\left\{z \leq f\left(X_{r-}^{N, i}\right)\right\}}\left[\varphi\left(0, X_{r-}^{N, j}+\frac{u}{\sqrt{N}}\right)-\varphi\left(X_{r-}^{N, i}, X_{r-}^{N, j}\right)\right] \pi^{i}(d r, d z, d u) \\
& +\int_{] s, t] \times \mathbb{R}_{+} \times \mathbb{R}} \mathbb{1}_{\left\{z \leq f\left(X_{r-}^{N, j}\right)\right\}}\left[\varphi\left(X_{r-}^{N, i}+\frac{u}{\sqrt{N}}, 0\right)-\varphi\left(X_{r-}^{N, i}, X_{r-}^{N, j}\right)\right] \pi^{j}(d r, d z, d u) \\
& \quad+\sum_{\substack{k=1 \\
k \notin\{i, j\}}}^{N} \int_{] s, t] \times \mathbb{R}_{+} \times \mathbb{R}} \mathbb{1}_{\left\{z \leq f\left(X_{r-}^{N, k}\right)\right\}}\left[\varphi\left(X_{r-}^{N, i}+\frac{u}{\sqrt{N}}, X_{r-}^{N, j}+\frac{u}{\sqrt{N}}\right)\right. \\
& \left.-\varphi\left(X_{r-}^{N, i}, X_{r-}^{N, j}\right)\right] \pi^{k}(d r, d z, d u) .
\end{aligned}
$$

We use the notation $\tilde{\pi}^{j}(d r, d z, d u)=\pi^{j}(d r, d z, d u)-d r d z \nu(d u)$ and set

$$
\begin{aligned}
& M_{s, t}^{N, i, j, 1}:=\int_{] s, t] \times \mathbb{R}_{+} \times \mathbb{R}} \mathbb{1}_{\left\{z \leq f\left(X_{r-}^{N, i}\right)\right\}}\left[\varphi\left(0, X_{r-}^{N, j}+\frac{u}{\sqrt{N}}\right)-\varphi\left(X_{r-}^{N, i}, X_{r-}^{N, j}\right)\right] \tilde{\pi}^{i}(d r, d z, d u), \\
& M_{s, t}^{N, i, j, 2}:=\int_{] s, t] \times \mathbb{R}_{+} \times \mathbb{R}} \mathbb{1}_{\left\{z \leq f\left(X_{r-}^{N, j}\right)\right\}}\left[\varphi\left(X_{r-}^{N, i}+\frac{u}{\sqrt{N}}, 0\right)-\varphi\left(X_{r-}^{N, i}, X_{r-}^{N, j}\right)\right] \tilde{\pi}^{j}(d r, d z, d u), \\
& W_{s, t}^{N, i, j}:=\sum_{\substack{k=1 \\
j \notin\{i, j\}}}^{N} \int_{] s, t] \times \mathbb{R}_{+} \times \mathbb{R}} \mathbb{1}_{\left\{z \leq f\left(X_{r-}^{N, k}\right)\right\}}\left[\varphi\left(X_{r-}^{N, i}+\frac{u}{\sqrt{N}}, X_{r-}^{N, j}+\frac{u}{\sqrt{N}}\right)\right. \\
& \left.-\varphi\left(X_{r-}^{N, i}, X_{r-}^{N, j}\right)\right] \tilde{\pi}^{k}(d r, d z, d u), \\
& \Delta_{s, t}^{N, i, j, 1}:=\int_{s}^{t} \int_{\mathbb{R}} f\left(X_{r}^{N, i}\right)\left[\varphi\left(0, X_{r}^{N, j}+\frac{u}{\sqrt{N}}\right)-\varphi\left(0, X_{r}^{N, j}\right)\right] d \nu(u) d r \\
& \Delta_{s, t}^{N, i, j, 2}:=\int_{s}^{t} \int_{\mathbb{R}} f\left(X_{r}^{N, j}\right)\left[\varphi\left(X_{r}^{N, i}+\frac{u}{\sqrt{N}}, 0\right)-\varphi\left(X_{r}^{N, i}, 0\right)\right] d \nu(u) d r, \\
& \Gamma_{s, t}^{N, i, j}:=\sum_{\substack{k=1 \\
k \notin\{i, j\}}}^{N} \int_{s}^{t} \int_{\mathbb{R}} f\left(X_{r}^{N, k}\right)\left[\varphi\left(X_{r}^{N, i}+\frac{u}{\sqrt{N}}, X_{r}^{N, j}+\frac{u}{\sqrt{N}}\right)-\varphi\left(X_{r}^{N, i}, X_{r}^{N, j}\right)\right. \\
& \left.-\frac{u}{\sqrt{N}} \partial_{x^{1}} \varphi\left(X_{r}^{N, i}, X_{r}^{N, j}\right)-\frac{u}{\sqrt{N}} \partial_{x^{2}} \varphi\left(X_{r}^{N, i}, X_{r}^{N, j}\right)\right] d \nu(u) d r \\
& -\int_{s}^{t} \int_{\mathbb{R}} \frac{u^{2}}{2} \partial^{2} \varphi\left(X_{r}^{N, i}, X_{r}^{N, j}\right) \frac{1}{N} \sum_{\substack{k=1 \\
k \notin\{i, j\}}}^{N} f\left(X_{r}^{N, k}\right) d \nu(u) d r \\
& R_{s, t}^{N, i, j}:=\frac{\sigma^{2}}{2} \int_{s}^{t} \partial^{2} \varphi\left(X_{r}^{N, i}, X_{r}^{N, j}\right)\left(\frac{1}{N} \sum_{\substack{k=1 \\
k \notin\{i, j\}}}^{N} f\left(X_{r}^{N, k}\right)-\frac{1}{N} \sum_{k=1}^{N} f\left(X_{r}^{N, k}\right)\right) d r
\end{aligned}
$$

where the two terms of the second line in the expression of $\Gamma_{s, t}^{N, i}$ can be artificially introduced since $\int_{\mathbb{R}} u d \nu(u)=0$. 


\section{Conditional propagation of chaos}

Finally, for $i=j$, we have

$$
\begin{aligned}
\varphi\left(X_{t}^{N, i}, X_{t}^{N, i}\right)=\varphi\left(X_{s}^{N, i}, X_{s}^{N, i}\right) \\
\quad-\alpha \int_{s}^{t} X_{r}^{N, i} \partial_{x^{1}} \varphi\left(X_{r}^{N, i}, X_{r}^{N, i}\right) d r-\alpha \int_{s}^{t} X_{r}^{N, i} \partial_{x^{2}} \varphi\left(X_{r}^{N, i}, X_{r}^{N, i}\right) d r \\
+\int_{] s, t] \times \mathbb{R}_{+} \times \mathbb{R}} \mathbb{1}_{\left\{z \leq f\left(X_{r-}^{N, i}\right)\right\}}\left[\varphi(0,0)-\varphi\left(X_{r-}^{N, i}, X_{r-}^{N, i}\right)\right] \pi^{i}(d r, d z, d u) \\
+\sum_{\substack{k=1 \\
k \neq i}}^{N} \int_{s s, t] \times \mathbb{R}_{+} \times \mathbb{R}} \mathbb{1}_{\left\{z \leq f\left(X_{r-}^{N, k}\right)\right\}}\left[\varphi\left(X_{r-}^{N, i}+\frac{u}{\sqrt{N}}, X_{r-}^{N, i}+\frac{u}{\sqrt{N}}\right)-\varphi\left(X_{r-}^{N, i}, X_{r-}^{N, i}\right)\right] \\
\pi^{k}(d r, d z, d u) .
\end{aligned}
$$

The associated martingales and error terms are given by

$$
\begin{aligned}
& M_{s, t}^{N, i}:= \int_{] s, t] \times \mathbb{R}_{+} \times \mathbb{R}} \mathbb{1}_{\left\{z \leq f\left(X_{r-}^{N, i}\right)\right\}}\left[\varphi(0,0)-\varphi\left(X_{r-}^{N, i}, X_{r-}^{N, i}\right)\right] \tilde{\pi}^{i}(d r, d z, d u), \\
& W_{s, t}^{N, i}:= \sum_{\substack{k=1 \\
k \neq i}} \int_{] s, t] \times \mathbb{R}_{+} \times \mathbb{R}} \mathbb{1}_{\left\{z \leq f\left(X_{r-}^{N, k}\right)\right\}}\left[\varphi\left(X_{r-}^{N, i}+\frac{u}{\sqrt{N}}, X_{r-}^{N, i}+\frac{u}{\sqrt{N}}\right)\right. \\
& \Delta_{s, t}^{N, i}:= \int_{s}^{t} \int_{\mathbb{R}} f\left(X_{r}^{N, i}\right)\left[\varphi(0,0)-\varphi\left(0, X_{r}^{N, i}, X_{r-}^{N, i}\right)\right] \tilde{\pi}^{k}(d r, d z, d u), \\
& \Gamma_{s, t}^{N, i}:= \sum_{\substack{k=1 \\
k \neq i}}^{N} \int_{s}^{t} \int_{\mathbb{R}} f\left(X_{r}^{N, k}\right)\left[\varphi\left(X_{r}^{N, i}, 0\right)+\varphi\left(X_{r}^{N, i}, X_{r}^{N, i}\right)\right] d \nu(u) d r, \\
&-\frac{u}{\sqrt{N}} \partial_{x^{1}} \varphi\left(X_{r}^{N, i}, X_{r}^{N, i}+\frac{u}{\sqrt{N}}\right)-\varphi\left(X_{r}^{N, i}, X_{r}^{N, i}\right) \\
&\left.-\int_{s}^{t} \int_{\mathbb{R}} \frac{u}{\sqrt{N}} \partial_{x^{2}}{ }^{2} \partial^{2} \varphi\left(X_{r}^{N, i}, X_{r}^{N, i}\right)\right] d \nu(u) d r \\
& R_{s, t}^{N, i}:= \frac{\sigma^{2}}{2} \int_{s}^{t} \partial^{2} \varphi\left(X_{r}^{N, i}, X_{r}^{N, i}\right) \frac{1}{N} \sum_{\substack{k=1 \\
k \neq i}}^{N} f\left(X_{r}^{N, k}\right) d \nu(u) d r, \\
&\left.\frac{1}{N} \sum_{\substack{k=1 \\
k \neq i}}^{N} f\left(X_{r}^{N, k}\right)-\frac{1}{N} \sum_{k=1}^{N} f\left(X_{r}^{N, k}\right)\right) d r,
\end{aligned}
$$

Then, since $\int_{\mathbb{R}} u d \nu(u)=0$, we obtain

$$
\begin{aligned}
F\left(\mu^{N}\right)= & \psi_{1}\left(\mu_{s_{1}}^{N}(f)\right) \ldots \psi_{k}\left(\mu_{s_{k}}^{N}(f)\right) \frac{1}{N^{2}} \sum_{i, j=1, i \neq j}^{N} \varphi_{1}\left(X_{s_{1}}^{N, i}, X_{s_{1}}^{N, j}\right) \ldots \varphi_{k}\left(X_{s_{k}}^{N, i}, X_{s_{k}}^{N, j}\right) \\
& {\left[M_{s, t}^{N, i, j, 1}+M_{s, t}^{N, i, j, 2}+W_{s, t}^{N, i, j}+\Delta_{s, t}^{N, i, j, 1}+\Delta_{s, t}^{N, i, j, 2}+\Gamma_{s, t}^{N, i, j}+R_{s, t}^{N, i, j}\right] } \\
& +\psi_{1}\left(\mu_{s_{1}}^{N}(f)\right) \ldots \psi_{k}\left(\mu_{s_{k}}^{N}(f)\right) \frac{1}{N^{2}} \sum_{i=1}^{N} \varphi_{1}\left(X_{s_{1}}^{N, i}, X_{s_{1}}^{N, i}\right) \ldots \varphi_{k}\left(X_{s_{k}}^{N, i}, X_{s_{k}}^{N, i}\right) \\
& {\left[M_{s, t}^{N, i}+W_{s, t}^{N, i}+\Delta_{s, t}^{N, i}+\Gamma_{s, t}^{N, i}+R_{s, t}^{N, i}\right] . }
\end{aligned}
$$

Using exchangeability and the boundedness of the $\varphi_{j}, \psi_{j}(1 \leq j \leq k)$ and the fact that 
$M^{N, i, j, 1}, \ldots, W^{N, i}$ are martingales, this implies

$$
\left|\mathbb{E}\left[F\left(\mu^{N}\right)\right]\right| \leq C \mathbb{E}\left[\left|\Delta_{s, t}^{N, i, j, 1}\right|+\left|\Delta_{s, t}^{N, i, j, 2}\right|+\left|\Gamma_{s, t}^{N, i, j}\right|+\left|R_{s, t}^{N, i, j}\right|+\frac{\left|\Delta_{s, t}^{N, i}\right|+\left|\Gamma_{s, t}^{N, i}\right|+\left|R_{s, t}^{N, i}\right|}{N}\right]
$$

Since $f$ is bounded and $\varphi \in C_{b}^{3}\left(\mathbb{R}^{2}\right)$, Taylor-Lagrange's inequality implies then that

$$
\left|\mathbb{E}\left[F\left(\mu^{N}\right)\right]\right| \leq \frac{C}{\sqrt{N}}
$$

Finally, using that $F$ is bounded and almost surely continuous at $\mu$ (see Step 1), we have

$$
\mathbb{E}[F(\mu)]=\lim _{N \rightarrow \infty} \mathbb{E}\left[F\left(\mu^{N}\right)\right]=0
$$

concluding our proof.

Let us end this section with the

Proof of Theorem 2.13. According to Proposition 3.1, the sequence $\left(\mu^{N}\right)_{N}$ is tight. Besides, thanks to Theorem 3.5, any limit $Q$ of a converging subsequence of $\left(\mathcal{L}\left(\mu^{N}\right)\right)_{N}$ is solution to the martingale problem.

By Theorem 3.4, there is a unique such distribution $Q$ which can be written as $Q=\mathcal{L}(\mu)$, with $\mu=\mathcal{L}\left(\bar{X}^{1} \mid \mathcal{W}\right)$, where $\left(\bar{X}^{j}\right)_{j \geq 1}$ is solution of (2.2). This implies the result.

\section{Proofs}

\subsection{A priori estimates}

In this subsection, we prove useful a priori upper bounds on some moments of the solutions of the SDEs (2.1) and (2.4).

Lemma 4.1. Suppose that Assumption 2.2 holds and that $f$ is bounded. Then

(i) for all $t>0, \sup _{N \in \mathbb{N}^{*} 0 \leq s \leq t} \sup _{0 \leq s} \mathbb{E}\left[\left(X_{s}^{N, 1}\right)^{2}\right]<+\infty$,

(ii) for all $t>0, \sup _{N \in \mathbb{N}^{*}} \mathbb{E}\left[\sup _{0 \leq s \leq t}\left|X_{s}^{N, 1}\right|\right]<+\infty$.

Proof. Step 1: Let us prove $(i)$.

$$
\begin{aligned}
& \left(X_{t}^{N, 1}\right)^{2}=\left(X_{0}^{N, 1}\right)^{2}-2 \alpha \int_{0}^{t}\left(X_{s}^{N, 1}\right)^{2} d s-\int_{[0, t] \times \mathbb{R}_{+} \times \mathbb{R}}\left(X_{s}^{N, 1}\right)^{2} \mathbb{1}_{\left\{z \leq f\left(X_{s-}^{N, 1}\right)\right\}} d \pi^{j}(s, z, u) \\
& +\sum_{j=2}^{N} \int_{[0, t] \times \mathbb{R}_{+} \times \mathbb{R}}\left[\left(X_{s-}^{N, 1}+\frac{u}{\sqrt{N}}\right)^{2}-\left(X_{s-}^{N, 1}\right)^{2}\right] \mathbb{1}_{\left\{z \leq f\left(X_{s-}^{N, j}\right)\right\}} d \pi^{j}(s, z, u) \\
& \leq\left(X_{0}^{N, 1}\right)^{2}+\sum_{j=2}^{N} \int_{[0, t] \times \mathbb{R}_{+} \times \mathbb{R}}\left[\left(X_{s-}^{N, 1}+\frac{u}{\sqrt{N}}\right)^{2}-\left(X_{s-}^{N, 1}\right)^{2}\right] \mathbb{1}_{\left\{z \leq f\left(X_{s-}^{N, j}\right)\right\}} d \pi^{j}(s, z, u) .
\end{aligned}
$$

As $f$ is bounded, and since $\int_{\mathbb{R}} u d \nu(u)=0$ and $\int_{\mathbb{R}} u^{2} d \nu(u)=\sigma^{2}$,

$$
\mathbb{E}\left[\left(X_{t}^{N, 1}\right)^{2}\right] \leq \mathbb{E}\left[\left(X_{0}^{N, 1}\right)^{2}\right]+\frac{\sigma^{2}}{N} \sum_{j=2}^{N} \int_{0}^{t} \mathbb{E}\left[f\left(X_{s}^{N, j}\right)\right] d s \leq \mathbb{E}\left[\left(X_{0}^{N, 1}\right)^{2}\right]+\sigma^{2}\|f\|_{\infty} t
$$


Step 2: Now we prove $(i i)$.

$$
\begin{aligned}
\left|X_{t}^{N, 1}\right| \leq & \left|X_{0}^{N, 1}\right|+\alpha \int_{0}^{t}\left|X_{s}^{N, 1}\right| d s \\
& +\int_{[0, t] \times \mathbb{R}_{+} \times \mathbb{R}}\left|X_{s-}^{N, 1}\right| \mathbb{1}_{\left\{z \leq f\left(X_{s-}^{N, 1}\right)\right\}} d \pi^{1}(s, z, u)+\frac{1}{\sqrt{N}}\left|M_{t}^{N}\right|,
\end{aligned}
$$

where $M_{t}^{N}$ is the martingale $M_{t}^{N}=\sum_{j=2}^{N} \int_{[0, t] \times \mathbb{R}_{+} \times \mathbb{R}} u \mathbb{1}_{\left\{z \leq f\left(X_{s-}^{N, j}\right)\right\}} d \pi^{j}(s, z, u)$. Then

$$
\begin{array}{r}
\sup _{0 \leq s \leq t}\left|X_{s}^{N, 1}\right| \leq\left|X_{0}^{N, 1}\right|+\alpha \int_{0}^{t}\left|X_{s}^{N, 1}\right| d s+\int_{[0, t] \times \mathbb{R}_{+} \times \mathbb{R}}\left|X_{s-}^{N, 1}\right| \mathbb{1}_{\left\{z \leq f\left(X_{s-}^{N, 1}\right)\right\}} d \pi^{1}(s, z, u) \\
+\frac{1}{\sqrt{N}} \sup _{0 \leq s \leq t}\left|M_{s}^{N}\right| .
\end{array}
$$

To conclude the proof, it is now sufficient to notice that

$$
\frac{1}{\sqrt{N}} \mathbb{E}\left[\sup _{0 \leq s \leq t}\left|M_{s}^{N}\right|\right] \leq \mathbb{E}\left[\frac{1}{N}\left[M^{N}\right]_{t}\right]^{1 / 2}
$$

is uniformly bounded in $N$, since $f$ is bounded, and to use the point $(i)$ of the lemma.

Lemma 4.2. Suppose that $f$ is bounded and that $\int_{\mathbb{R}} x^{2} d \nu_{0}(x)<\infty$. Then any solution $\left(\bar{X}_{t}^{1}\right)_{t \geq 0}$ of (2.4) a priori satisfies $\mathbb{E}\left[\sup _{s \leq t}\left(\bar{X}_{s}^{1}\right)^{2}\right]<\infty$ for all $t \geq 0$.

Proof. We first prove the weaker result

$$
\sup _{s \leq t} \mathbb{E}\left[\left(\bar{X}_{s}^{1}\right)^{2}\right]<\infty .
$$

By Itô's formula,

$$
\begin{aligned}
\left(\bar{X}_{t}^{1}\right)^{2}=\left(\bar{X}_{0}^{1}\right)^{2}- & 2 \alpha \int_{0}^{t}\left(\bar{X}_{s}^{1}\right)^{2} d s+2 \sigma \int_{0}^{t} \bar{X}_{s}^{1} \sqrt{\mu_{s}(f)} d W_{s} \\
& +\sigma^{2} \int_{0}^{t} \mu_{s}(f) d s-\int_{[0, t] \times \mathbb{R}_{+}}\left(\bar{X}_{s-}^{1}\right)^{2} \mathbb{1}_{\left\{z \leq f\left(\bar{X}_{s-}^{1}\right)\right\}} d \pi(s, z) \\
\leq & \left(\bar{X}_{0}^{1}\right)^{2}+2 \sigma \int_{0}^{t} \bar{X}_{s}^{1} \sqrt{\mu_{s}(f)} d W_{s}+\sigma^{2} \int_{0}^{t} \mu_{s}(f) d s
\end{aligned}
$$

Introducing, for any $M>0, \tau_{M}:=\inf \left\{t>0:\left|\bar{X}_{t}^{1}\right|>M\right\}$ and $u_{M}(t):=\mathbb{E}\left[\left(\bar{X}_{t \wedge \tau_{M}}^{1}\right)^{2}\right]$, we have, for all $t \geq 0$,

$$
u_{M}(t) \leq \mathbb{E}\left[\left(\bar{X}_{0}^{1}\right)^{2}\right]+\sigma^{2}\|f\|_{\infty} t
$$

Then Grönwall's lemma implies that for all $T>0$,

$$
\sup _{M>0} \sup _{0 \leq t \leq T} u_{M}(t)<\infty .
$$

This implies that the stopping times $\tau_{M}$ tend to infinity as $M$ goes to infinity. (4.1) is a then a consequence of (4.3) and Fatou's lemma. Then, using Burkholder-Davis-Gundy inequality to control the martingale part in (4.2), we have, for all $t \geq 0$,

$$
\begin{aligned}
\mathbb{E}\left[\sup _{0 \leq s \leq t}\left(\bar{X}_{s}^{1}\right)^{2}\right] & \leq \mathbb{E}\left[\left(\bar{X}_{0}^{1}\right)^{2}\right]+\sigma^{2}\|f\|_{\infty} t+2 \sigma\|f\|_{\infty} \mathbb{E}\left[\left(\int_{0}^{t}\left(\bar{X}_{s}^{1}\right)^{2} d s\right)^{1 / 2}\right] \\
& \leq \mathbb{E}\left[\left(\bar{X}_{0}^{1}\right)^{2}\right]+\sigma^{2}\|f\|_{\infty} t+2 \sigma\|f\|_{\infty}\left(1+\int_{0}^{t} \mathbb{E}\left[\left(\bar{X}_{s}^{1}\right)^{2}\right] d s\right) d s .
\end{aligned}
$$

Then the result follows from point (4.1). 


\subsection{Well-posedness of the limit equation (2.4)}

Proof of Item 2. of Theorem 2.6. The proof is done using a classical Picard-iteration. For that sake we introduce the sequence of processes $\bar{X}_{t}^{[0]} \equiv \bar{X}_{0}$, and construct the process $\bar{X}^{[n+1]}$ from $\bar{X}^{[n]}$ and $\mu_{s}^{n}=P\left(\bar{X}_{s}^{[n]} \in \cdot \mid \mathcal{W}\right)$ in the following way:

- let $0<\tau_{1}<\tau_{2}<\ldots$ be the jump times of

$$
t \geq 0 \mapsto \int_{[0, t] \times \mathbb{R}_{+}} \mathbb{1}_{\left\{z \leq f\left(\bar{X}_{s-}^{[n]}\right)\right\}} \pi(d s, d z),
$$

- set $\bar{X}_{\tau_{i}}^{[n+1]}=0(i \geq 1)$ and define, $\bar{X}^{[n+1]}$ between the jump times: for all $i \geq 1$, for all $t \in] \tau_{i}, \tau_{i+1}[$,

$$
\bar{X}_{t}^{[n+1]}=\sigma \int_{0}^{t} e^{-\alpha(t-s)} \sqrt{\mu_{s}^{n}(f)} d W_{s} .
$$

One can note, using the integration by parts formula, that $\bar{X}^{[n+1]}$ satisfies the following SDE:

$$
\begin{aligned}
\bar{X}_{t}^{[n+1]}:= & \bar{X}_{0}-\alpha \int_{0}^{t} \bar{X}_{s}^{[n+1]} d s \\
& -\int_{[0, t] \times \mathbb{R}_{+} \times \mathbb{R}} \bar{X}_{s-}^{[n+1]} \mathbb{1}_{\left\{z \leq f\left(\bar{X}_{s-}^{[n]}\right)\right\}} \pi(d s, d z, d u)+\sigma \int_{0}^{t} \sqrt{\mu_{s}^{n}(f)} d W_{s} .
\end{aligned}
$$

Using the same proof as the one of Lemma 4.2, we can show that for all $t \geq 0$,

$$
\sup _{n \in \mathbb{N}} \sup _{0 \leq s \leq t} \mathbb{E}\left[\left(\bar{X}_{s}^{[n]}\right)^{2}\right]<+\infty \text {. }
$$

Now, we prove the convergence of $\bar{X}_{t}^{[n]}$. The same strategy as the one of the proof of Item 1. of Theorem 2.6 allows to show that

$$
\delta_{t}^{n}:=\mathbb{E} \sup _{s \leq t}\left|a\left(\bar{X}_{s}^{[n]}\right)-a\left(\bar{X}_{s}^{[n-1]}\right)\right| \text { satisfies } \delta_{t}^{n} \leq C(t+\sqrt{t}) \delta_{t}^{n-1},
$$

for all $n \geq 1$, for a constant $C$ only depending on the parameters of the model, but not on $n$, neither on $t$. Choose $t_{1}$ such that

$$
C\left(t_{1}+\sqrt{t_{1}}\right) \leq \frac{1}{2}
$$

Since $\sup _{s \leq t_{1}}\left|a\left(\bar{X}_{s}^{[0]}\right)\right|=a\left(\bar{X}_{0}\right) \leq\|a\|_{\infty}$, we deduce from this that

$$
\delta_{t_{1}}^{n} \leq 2^{-n}\|a\|_{\infty} .
$$

This implies the almost sure convergence of $a\left(\bar{X}_{t}^{[n]}\right)_{n}$ to some random variable $Z_{t}$ for all $t \in\left[0, t_{1}\right]$. As $a$ is an increasing function, the almost sure convergence of $\bar{X}_{t}^{[n]}$ to some (possibly infinite) random variable $\bar{X}_{t}$ follows from this. The almost sure finiteness of $\bar{X}_{t}$ is then guaranteed by Fatou's lemma and (4.4).

Now let us prove that $\bar{X}$ is solution of the limit equation (2.4) which follows by standard arguments (note that the jump term does not cause troubles because it is of finite activity). The most important point is to notice that

$$
\mu_{t}^{n}(f)=\mathbb{E}\left(f\left(\bar{X}_{t}^{[n]}\right) \mid \mathcal{W}\right) \rightarrow \mathbb{E}\left(f\left(\bar{X}_{t}\right) \mid \mathcal{W}\right)
$$

almost surely, which follows from the almost sure convergence of $f\left(\bar{X}_{t}^{[n]}\right) \rightarrow f\left(\bar{X}_{t}\right)$, using dominated convergence.

Once the convergence is proven on the time interval $\left[0, t_{1}\right]$, we can proceed iteratively over successive intervals $\left[k t_{1},(k+1) t_{1}\right]$ to conclude that $\bar{X}$ is solution of (2.4) on $\mathbb{R}_{+}$. Finally, (2.5) follows from Lemma 4.2. 


\subsection{Proof of Proposition 2.9}

We now give the

Proof of Proposition 2.9. ( $i$ ) Given a Brownian motion $W$ and i.i.d. Poisson measures $\pi^{i}$, the same proof as the one of Theorem 2.6 implies the existence and the uniqueness of the system given in (2.2) for $1 \leq i \leq N$.

(ii) The construction of the proof of Item 2. of Theorem 2.6, together with the proof of Theorem 1.1 of Chapter IV.1 and of Theorem 9.1 in Chapter IV.9 of [14], imply the existence of a measurable function $\Phi$ that does not depend on $k=1, \ldots, N$, and that satisfies, for each $1 \leq k \leq N$,

$$
\bar{X}^{k}=\Phi\left(\bar{X}_{0}^{k}, \pi^{k}, W\right)
$$

and for all $t \geq 0$,

$$
\bar{X}_{\mid[0, t]}^{k}=\Phi_{t}\left(\bar{X}_{0}^{k}, \pi_{\mid[0, t] \times \mathbb{R}_{+} \times \mathbb{R}}^{k},\left(W_{s}\right)_{s \leq t}\right) ;
$$

in other words, our process is non-anticipative and does only depend on the underlying noise up to time $t$.

Then we can write, for all continuous bounded functions $g, h$,

$$
\mathbb{E}\left[g\left(\bar{X}^{i}\right) h\left(\bar{X}^{j}\right) \mid \mathcal{W}\right]=\mathbb{E}\left[g\left(\Phi\left(\bar{X}_{0}^{i}, \pi^{i}, W\right)\right) h\left(\Phi\left(\bar{X}_{0}^{j}, \pi^{j}, W\right)\right) \mid \mathcal{W}\right]=\psi(W),
$$

where

$$
\begin{aligned}
\psi(w) & :=\mathbb{E}\left[g\left(\Phi\left(\bar{X}_{0}^{i}, \pi^{i}, w\right)\right) h\left(\Phi\left(\bar{X}_{0}^{j}, \pi^{j}, w\right)\right)\right] \\
& =\mathbb{E}\left[g\left(\Phi\left(\bar{X}_{0}^{i}, \pi^{i}, w\right)\right)\right] \mathbb{E}\left[h\left(\Phi\left(\bar{X}_{0}^{j}, \pi^{j}, w\right)\right)\right]=: \psi_{i}(w) \psi_{j}(w) .
\end{aligned}
$$

With the same reasoning, we show that $\mathbb{E}\left[g\left(\bar{X}^{i}\right) \mid \mathcal{W}\right]=\psi_{i}(W)$ and $\mathbb{E}\left[h\left(\bar{X}^{j}\right) \mid \mathcal{W}\right]=$ $\psi_{j}(W)$. The same arguments prove the mutual independence of $\bar{X}^{1}, \ldots \bar{X}^{N}$ conditionally to $W$.

(iii) Using the representation $\bar{X}_{\mid[0, t]}^{k}=\Phi_{t}\left(\bar{X}_{0}^{k}, \pi^{k}, W\right)$, we can write for any continuous and bounded function $g: D([0, t], \mathbb{R}) \rightarrow \mathbb{R}$,

$$
\int_{\mathbb{R}} g d\left(N^{-1} \sum_{i=1}^{N} \delta_{\bar{X}_{|| 0, t]}^{i}}\right)=\frac{1}{N} \sum_{i=1}^{N} g\left(\bar{X}_{\mid[0, t]}^{i}\right)=\frac{1}{N} \sum_{i=1}^{N} g \circ \Phi_{t}\left(\bar{X}_{0}^{i}, \pi^{i}, W\right) .
$$

Using the law of large numbers on the account of the sequence of i.i.d. PRM's and working conditionally on $W$, we obtain that

$$
\begin{aligned}
\lim _{N \rightarrow \infty} \int_{\mathbb{R}} g d\left(N^{-1} \sum_{i=1}^{N} \delta_{\left.\bar{X}_{\mid[0, t]}^{i}\right)}\right) & =\mathbb{E}\left[g \circ \Phi_{t}\left(\bar{X}_{0}^{1}, \pi^{1}, W\right) \mid \mathcal{W}\right] \\
& =\mathbb{E}\left[g\left(\bar{X}_{\mid[0, t]}^{1}\right) \mid \mathcal{W}\right]=\mathbb{E}\left[g\left(\bar{X}_{\mid[0, t]}^{1}\right) \mid\left(W_{s}\right)_{s \leq t}\right],
\end{aligned}
$$

where we have used (4.5).

\subsection{Proof of Theorem 2.11}

We are finally able to give the

Proof of Theorem 2.11. Step 1: Let us begin by proving that any solution $\left(\bar{X}^{i}\right)_{i \geq 1}$ of (2.2) is solution of (2.7).

By Proposition 2.9.(ii), conditionally to $\mathcal{W}$, the variables $\bar{X}_{t}^{i}$ are i.i.d. This implies that the directing measure of $\left(\bar{X}_{t}^{i}\right)_{i \geq 1}$ is $\mathcal{L}\left(\bar{X}_{t}^{i} \mid \mathcal{W}\right)$ (see Lemma (2.12) of [1]). 
Step 2. It is now sufficient to prove that $\left(\bar{X}^{i}\right)_{i \geq 1}$ is the only solution of (2.7) defined w.r.t. the same Brownian motion, Poisson random measures and initial conditions. For that sake, let us consider $\left(\bar{Y}^{i}\right)_{i \geq 1}$ any solution of (2.7), and prove that $\left(\bar{X}^{i}\right)_{i \geq 1}=\left(\bar{Y}^{i}\right)_{i \geq 1}$ almost surely. In the rest of the proof, $\mu_{t}$ denotes only the directing measure of the system $\left(\bar{Y}_{t}^{i}\right)_{i \geq 1}$. So we want to prove that $\mu_{t}(f):=\mathbb{E}\left[f\left(\bar{Y}_{t}^{1}\right) \mid \mu\right]=\mathbb{E}\left[f\left(\bar{Y}_{t}^{1}\right) \mid \mathcal{W}\right]$ a.s..

To begin with, Lemma (2.15) of [1] implies that $\mu_{t}(f)$ is the almost sure limit of $N^{-1} \sum_{j=1}^{N} f\left(\bar{Y}_{t}^{j}\right)$. We now prove that this sequence converges to $\mathbb{E}\left[f\left(\bar{Y}_{t}^{1}\right) \mid \mathcal{W}\right]$. For this purpose, we introduce an auxiliary system $\left(\widetilde{X}^{N, i}\right)_{1 \leq i \leq N}$, driven by the same Brownian motion $W$ and the same Poisson random measures $\pi^{i}$, with $\bar{Y}_{0}^{i}=\tilde{X}_{0}^{N, i}(i \geq 1)$, replacing the term $\mu_{t}(f)$ by the empirical measure:

$d \widetilde{X}_{t}^{N, i}=-\alpha \widetilde{X}_{t}^{N, i} d t+\sqrt{\frac{1}{N} \sum_{j=1}^{N} f\left(\widetilde{X}_{t}^{N, j}\right)} d W_{t}-\widetilde{X}_{t-}^{N, i} \int_{\mathbb{R}_{+}} \mathbb{1}_{\left\{z \leq f\left(\widetilde{X}_{t-}^{N, i}\right)\right\}} \pi^{i}(d t, d z), \widetilde{X}_{0}^{N, i}=\bar{Y}_{0}^{i}$.

Notice that $\left(\bar{X}^{i}\right)_{i \geq 1},\left(\bar{Y}^{i}\right)_{i \geq 1}$ and $\left(\tilde{X}^{N, i}\right)_{1 \leq i \leq N}$ are all defined on the same probability space, driven by the same Brownian motion $W$ and the same Poisson random measures $\pi^{i}$.

It is now sufficient to prove that both for $\left(\bar{Y}^{i}\right)_{i \geq 1}$ and for $\left(\bar{X}^{i}\right)_{i \geq 1}$,

$$
\mathbb{E}\left[\left|a\left(\bar{Y}_{t}^{i}\right)-a\left(\widetilde{X}_{t}^{N, i}\right)\right|\right]+\mathbb{E}\left[\left|a\left(\bar{X}_{t}^{i}\right)-a\left(\widetilde{X}_{t}^{N, i}\right)\right|\right] \leq C_{t} N^{-1 / 2} .
$$

Indeed, suppose we have already proven the above control (4.6). Then

$$
\begin{aligned}
\mathbb{E}\left[\left|\frac{1}{N} \sum_{j=1}^{N} f\left(\bar{Y}_{t}^{j}\right)-\mathbb{E}\left[f\left(\bar{X}_{t}^{1}\right) \mid \mathcal{W}\right]\right|\right] & \leq \frac{1}{N} \sum_{j=1}^{N} \mathbb{E}\left[\left|f\left(\bar{Y}_{t}^{j}\right)-f\left(\widetilde{X}_{t}^{N, j}\right)\right|\right] \\
+ & \frac{1}{N} \sum_{j=1}^{N} \mathbb{E}\left[\left|f\left(\widetilde{X}_{t}^{N, j}\right)-f\left(\bar{X}_{t}^{j}\right)\right|\right]+\mathbb{E}\left[\left|\frac{1}{N} \sum_{j=1}^{N} f\left(\bar{X}_{t}^{j}\right)-\mathbb{E}\left[f\left(\bar{X}_{t}^{1}\right) \mid \mathcal{W}\right]\right|\right] .
\end{aligned}
$$

Then, (4.6) and Assumption 2.3 imply that the first and the second term of the sum above are smaller than $C_{t} N^{-1 / 2}$ for some $C_{t}>0$. In addition, by item (ii) of Proposition 2.9, the variables $\left(\bar{X}^{j}\right)_{1 \leq j \leq N}$ are i.i.d., conditionally on $W$. Consequently, and since $f$ is bounded, the third term is smaller than $C_{t} N^{-1 / 2}$.

The above implies that, as $N \rightarrow \infty, \frac{1}{N} \sum_{j=1}^{N} f\left(\bar{Y}_{t}^{j}\right)$ converges in $L^{1}(P)$ to $\mathbb{E}\left[f\left(\bar{X}_{t}^{1}\right) \mid \mathcal{W}\right]$. On the other hand, we know this sequence converges almost surely to $\mu_{t}(f)$. Thus,

$$
\mathbb{E}\left[f\left(\bar{Y}_{t}^{1}\right) \mid \mu\right]=\mu_{t}(f)=\mathbb{E}\left[f\left(\bar{X}_{t}^{1}\right) \mid \mathcal{W}\right]=\mathbb{E}\left[f\left(\bar{X}_{t}^{i}\right) \mid \mathcal{W}\right] \text { a.s.. }
$$

As a consequence, $\left(\bar{Y}^{i}\right)_{i \geq 1}$ is solution of the infinite system

$$
d \bar{Y}_{t}^{i}=-\alpha \bar{Y}_{t}^{i} d t+\sigma \sqrt{\mathbb{E}\left[f\left(\bar{X}_{t}^{i}\right) \mid \mathcal{W}\right]} d W_{t}-\bar{Y}_{t-}^{i} \int_{\mathbb{R}_{+}} \mathbb{1}_{\left\{z \leq f\left(\bar{Y}_{t-}^{i}\right)\right\}} \pi^{i}(d t, d z),
$$

while $\left(\bar{X}^{i}\right)_{i \geq 1}$ in (2.2) is solution of

$$
d \bar{X}_{t}^{i}=-\alpha \bar{X}_{t}^{i} d t+\sigma \sqrt{\mathbb{E}\left[f\left(\bar{X}_{t}^{i}\right) \mid \mathcal{W}\right]} d W_{t}-\bar{X}_{t-}^{i} \int_{\mathbb{R}_{+}} \mathbb{1}_{\left\{z \leq f\left(\bar{X}_{t-}^{i}\right)\right\}} \pi^{i}(d t, d z),
$$

with $\bar{X}_{0}^{i}=\bar{Y}_{0}^{i}$, for all $i \geq 1$.

Step 3. In the previous step, we have proved that $\mathbb{E}\left[f\left(\bar{Y}_{t}^{i}\right) \mid \mu\right]=\mathbb{E}\left[f\left(\bar{X}_{t}^{i}\right) \mid \mathcal{W}\right]$. So, we still have not proved that $\bar{Y}^{i}$ satisfies the SDE (2.2), which would have allowed to conclude that $\bar{Y}=\bar{X}$ almost surely (by Theorem 2.6.(i)). We prove it in this step. 
For that sake, consider $\tau_{M}=\inf \left\{t>0:\left|\bar{X}_{t}^{i}\right| \wedge\left|\bar{Y}_{t}^{i}\right|>M\right\}$ for $M>0$. We prove that $\mathbb{E}\left[\left|\bar{X}_{t \wedge \tau_{M}}^{i}-\bar{Y}_{t \wedge \tau_{M}}^{i}\right|\right]=0$ for all $M>0$. Recalling Lemma 4.2 and the fact that we can prove a similar control for $\bar{Y}^{i}$ this implies, by Fatou's lemma, that $\mathbb{E}\left[\left|\bar{X}_{t}^{i}-\bar{Y}_{t}^{i}\right|\right]=0$.

Let $u_{M}(t):=\mathbb{E}\left[\left|\bar{X}_{t \wedge \tau_{M}}^{i}-\bar{Y}_{t \wedge \tau_{M}}^{i}\right|\right]$. To see that $u_{M}(t)=0$, it is sufficient to apply Grönwall's lemma to the following inequality

$$
u_{M}(t) \leq \alpha \int_{0}^{t} u_{M}(s) d s+\mathbb{E}\left[\int_{\left[0, t \wedge \tau_{M}\right] \times \mathbb{R}_{+}}\left|\bar{X}_{s-}^{i} \mathbb{1}_{\left\{z \leq f\left(\bar{X}_{s-}^{i}\right)\right\}}-\bar{Y}_{s-}^{i} \mathbb{1}_{\left\{z \leq f\left(\bar{Y}_{s-}^{i}\right)\right\}}\right| \pi^{i}(d s, d z)\right]
$$

implying that

$$
\begin{aligned}
u_{M}(t) \leq & \alpha \int_{0}^{t} u_{M}(s) d s+\mathbb{E}\left[\int_{\left[0, t \wedge \tau_{M}\right] \times \mathbb{R}_{+}} \mathbb{1}_{\left\{z \in\left[0, f\left(\bar{X}_{s-}^{i}\right) \wedge f\left(\bar{Y}_{s-}^{i}\right)\right]\right\}}\left|\bar{X}_{s-}^{i}-\bar{Y}_{s-}^{i}\right| \pi^{i}(d s, d z)\right] \\
& +\mathbb{E}\left[\int_{\left[0, t \wedge \tau_{M}\right] \times \mathbb{R}_{+}} \mathbb{1}_{\left.\left.\{z \in] f\left(\bar{X}_{s-}^{i}\right) \wedge f\left(\bar{Y}_{s-}^{i}\right), f\left(\bar{X}_{s-}^{i}\right) \vee f\left(\bar{Y}_{s-}^{i}\right)\right]\right\}}\left|\bar{X}_{s-}^{i}\right| \vee\left|\bar{Y}_{s-}^{i}\right| \pi^{i}(d s, d z)\right],
\end{aligned}
$$

whence

$$
u_{M}(t) \leq C(1+M) \int_{0}^{t} u_{M}(s) d s
$$

and thus $u_{M}(t)=0$.

Hence $\left(\bar{Y}^{i}\right)_{i \geq 1}$ is solution of the infinite system (2.2) and $\mu=\mathcal{L}\left(\bar{Y}^{1} \mid \mathcal{W}\right)$, its directing measure, is uniquely determined.

Step 4. Finally, let us show (4.6). We only prove it for $\bar{Y}^{i}$, the proof for $\bar{X}^{i}$ is similar. By exchangeability, it is sufficient to work with $\bar{Y}^{1}$. We decompose the evolution of $a\left(\bar{Y}_{t}^{1}\right)$ in the following way.

$$
\begin{aligned}
a\left(\bar{Y}_{t}^{1}\right) & =a\left(\bar{Y}_{0}^{1}\right)-\alpha \int_{0}^{t} a^{\prime}\left(\bar{Y}_{s}^{1}\right) \bar{Y}_{s}^{1} d s+\int_{[0, t] \times \mathbb{R}_{+}}\left(a(0)-a\left(\bar{Y}_{s-}^{1}\right)\right) \mathbb{1}_{\left\{z \leq f\left(\bar{Y}_{s-}^{1}\right)\right\}} \pi^{1}(d s, d z) \\
& +\frac{\sigma^{2}}{2} \int_{0}^{t} a^{\prime \prime}\left(\bar{Y}_{s}^{1}\right) \frac{1}{N} \sum_{j=1}^{N} f\left(\bar{Y}_{s}^{j}\right) d s-B_{t}^{N}+\sigma \int_{0}^{t} a^{\prime}\left(\bar{Y}_{s}^{1}\right) \sqrt{\frac{1}{N} \sum_{j=1}^{N} f\left(\bar{Y}_{s}^{j}\right)} d W_{s}-M_{t}^{N}
\end{aligned}
$$

where

$$
B_{t}^{N}=\frac{\sigma^{2}}{2} \int_{0}^{t} a^{\prime \prime}\left(\bar{Y}_{s}^{1}\right)\left(\frac{1}{N} \sum_{j=1}^{N} f\left(\bar{Y}_{s}^{j}\right)-\mathbb{E}\left[f\left(\bar{Y}_{s}^{1}\right) \mid \mu\right]\right) d s
$$

and

$$
M_{t}^{N}=\sigma \int_{0}^{t} a^{\prime}\left(\bar{Y}_{s}^{1}\right)\left(\sqrt{\frac{1}{N} \sum_{j=1}^{N} f\left(\bar{Y}_{s}^{j}\right)}-\sqrt{\mathbb{E}\left[f\left(\bar{Y}_{s}^{1}\right) \mid \mu\right]}\right) d W_{s}
$$

Clearly,

$$
<M^{N}>_{t} \leq \sigma^{2}\left(\sup _{x \in \mathbb{R}}\left|a^{\prime}(x)^{2}\right|\right) \int_{0}^{t}\left(\sqrt{\frac{1}{N} \sum_{j=1}^{N} f\left(\bar{Y}_{s}^{j}\right)}-\sqrt{\mathbb{E}\left[f\left(\bar{Y}_{s}^{1}\right) \mid \mu\right]}\right)^{2} d s .
$$

Recall that the variables $\bar{Y}_{s}^{j}(1 \leq j \leq N)$ are i.i.d. conditionally to $\mu$. Hence we may take conditional expectation $\mathbb{E}(\cdot \mid \mu)$ and use the fact that $f$ is lower bounded such that $\sqrt{ } \cdot$ is Lipschitz on [inf $f, \infty[$ implying

$$
\left(\sqrt{\frac{1}{N} \sum_{j=1}^{N} f\left(\bar{Y}_{s}^{j}\right)}-\sqrt{\mathbb{E}\left[f\left(\bar{Y}_{s}^{1}\right) \mid \mu\right]}\right)^{2} \leq C\left(\frac{1}{N} \sum_{j=1}^{N} f\left(\bar{Y}_{s}^{j}\right)-\mathbb{E}\left[f\left(\bar{Y}_{s}^{1}\right) \mid \mu\right]\right)^{2}
$$


to deduce that

$$
\mathbb{E}\left[<M^{N}>_{t}\right] \leq C_{t} N^{-1} \text { and } \mathbb{E}\left[B_{t}^{N}\right] \leq C_{t} N^{-1} .
$$

Then, applying Itô's formula on $a\left(\tilde{X}^{N, 1}\right)$, we obtain the same equation as (4.7), but without the terms $B_{t}^{N}$ and $M_{t}^{N}$. Introducing

$$
u(t):=\sup _{0 \leq s \leq t} \mathbb{E}\left[\left|a\left(\bar{Y}_{s}^{1}\right)-a\left(\tilde{X}_{s}^{N, 1}\right)\right|\right],
$$

we can prove with the same reasoning as in the proof of Theorem 2.6 that

$$
u(t) \leq C(1+t) u(t)+\frac{C_{t}}{\sqrt{N}},
$$

where $C$ and $C_{t}$ are independent of $N$. Finally, using the arguments of the proof of Theorem 2.6, this implies (4.6).

\section{References}

[1] David Aldous, Exchangeability and related topics, Ecole d'Eté de Probabilités de Saint-Flour: XIII - 1983, Lecture notes in mathematics, no. 1117, Springer-Verlag ed., 1983. MR-0883646

[2] René Carmona, François Delarue, and Daniel Lacker, Mean field games with common noise, The Annals of Probability 44 (2016), no. 6, 3740-3803. MR-3572323

[3] Michele Coghi and Franco Flandoli, Propagation of chaos for interacting particles subject to environmental noise, The Annals of Applied Probability 26 (2016), no. 3, 1407-1442 (EN). MR-3513594

[4] Quentin Cormier, Etienne Tanré, and Romain Veltz, Long time behavior of a mean-field model of interacting neurons, arXiv:1810.08562 [math] (2019). MR-4080722

[5] A. De Masi, A. Galves, E. Löcherbach, and E. Presutti, Hydrodynamic Limit for Interacting Neurons, Journal of Statistical Physics 158 (2015), no. 4, 866-902 (en). MR-3311484

[6] Sylvain Delattre, Nicolas Fournier, and Marc Hoffmann, Hawkes processes on large networks, The Annals of Applied Probability 26 (2016), no. 1, 216-261. MR-3449317

[7] Azzouz Dermoune, Propagation and conditional propagation of chaos for pressureless gas equations, Probability Theory and Related Fields 126 (2003), no. 4, 459-476 (en). MR2001194

[8] Susanne Ditlevsen and Eva Löcherbach, Multi-class Oscillating Systems of Interacting Neurons, Stochastic Processes and their Applications 127 (2017), no. 6, 1840-1869. MR-3646433

[9] Xavier Erny, Eva Löcherbach, and Dasha Loukianova, Mean field limits for interacting Hawkes processes in a diffusive regime, arXiv:1904.06985 [math] (2019). MR-3772474

[10] Nicolas Fournier and Eva Löcherbach, On a toy model of interacting neurons, Annales de l'Institut Henri Poincaré - Probabilités et Statistiques 52 (2016), 1844-1876. MR-3573298

[11] A. Galves and E. Löcherbach, Infinite systems of interacting chains with memory of variable length - a stochastic model for biological neural nets, Journal of Statistical Physics 151 (2013), no. 5, 896-921. MR-3055382

[12] Carl Graham, McKean-Vlasov Ito-Skorohod equations, and nonlinear diffusions with discrete jump sets, Stochastic Processes and their Applications 40 (1992), no. 1, 69-82. MR-1145460

[13] Edwin Hewitt and Leonard J. Savage, Symmetric measures on Cartesian products, Transactions of the American Mathematical Society 80 (1955), no. 2, 470-501 (en). MR-0076206

[14] Nobuyuki Ikeda and Shinzo Watanabe, Stochastic Differential Equations and Diffusion Processes, second ed., North-Holland Publishing Company, 1989. MR-0637061

[15] Jean Jacod and Albert N Shiryaev, Limit Theorems for Stochastic Processes, second ed., Springer-Verlag BerlinHeidelberg NewYork, 2003. MR-1943877

[16] Alain-Sol Sznitman, Topics in propagation of chaos, Ecole d'Eté de Probabilités de Saint-Flour: XIX - 1989, Lecture notes in mathematics, no. 1464, Springer, Berlin, 1989, OCLC: 23253880, pp. 167-251 (en). MR-1108185 
Acknowledgments. The authors thank an anonymous referee for useful remarks and very careful reading. X.E. thanks François Delarue for a very helpful discussion about the limit process that led to the statement of Theorem 2.11. This research has been conducted as part of the FAPESP project Research, Innovation and Dissemination Center for Neuromathematics(grant 2013/07699-0) and of the ANR project ANR-19-CE40-0024. 


\section{Electronic Journal of Probability Electronic Communications in Probability}

\section{Advantages of publishing in EJP-ECP}

- Very high standards

- Free for authors, free for readers

- Quick publication (no backlog)

- Secure publication $\left(\mathrm{LOCKSS}^{1}\right)$

- Easy interface (EJMS²)

\section{Economical model of EJP-ECP}

- Non profit, sponsored by $\mathrm{IMS}^{3}, \mathrm{BS}^{4}$, ProjectEuclid ${ }^{5}$

- Purely electronic

\section{Help keep the journal free and vigorous}

- Donate to the IMS open access fund ${ }^{6}$ (click here to donate!)

- Submit your best articles to EJP-ECP

- Choose EJP-ECP over for-profit journals

\footnotetext{
${ }^{1}$ LOCKSS: Lots of Copies Keep Stuff Safe http://www. lockss.org/

${ }^{2}$ EJMS: Electronic Journal Management System http://www.vtex.lt/en/ejms.html

${ }^{3}$ IMS: Institute of Mathematical Statistics http://www.imstat.org/

${ }^{4}$ BS: Bernoulli Society http://www. bernoulli-society.org/

${ }^{5}$ Project Euclid: https://projecteuclid.org/

${ }^{6}$ IMS Open Access Fund: http://www.imstat.org/publications/open.htm
} 